MATHEMATICS OF COMPUTATION

Volume 71, Number 237, Pages 1-25

S $0025-5718(01) 01318-7$

Article electronically published on May 21, 2001

\title{
MIXED FINITE ELEMENT METHODS FOR UNILATERAL PROBLEMS: CONVERGENCE ANALYSIS AND NUMERICAL STUDIES
}

\author{
PATRICE COOREVITS, PATRICK HILD, KHALID LHALOUANI, AND TAOUFIK SASSI
}

\begin{abstract}
In this paper, we propose and study different mixed variational methods in order to approximate with finite elements the unilateral problems arising in contact mechanics. The discretized unilateral conditions at the candidate contact interface are expressed by using either continuous piecewise linear or piecewise constant Lagrange multipliers in the saddle-point formulation. A priori error estimates are established and several numerical studies corresponding to the different choices of the discretized unilateral conditions are achieved.
\end{abstract}

\section{INTRODUCTION}

Mixed finite element studies for approximating Signorini problems (see [6]) and the corresponding vector-valued unilateral contact problems have been used by several authors (see [11, 14]). In the finite element approximation of the unilateral contact problem between elastic bodies, the discretized noninterpenetration conditions constitute the key point of the approximation model. In the mixed methods, the latter unilateral conditions (of the displacements) can be expressed by using either a piecewise constant or continuous piecewise linear Lagrange multipliers in the saddle-point formulation. Concerning the piecewise constant Lagrange multiplier approach, several error estimates have been derived in [1] and more recently in 17 .

Our first purpose is to carry out the convergence analysis and the a priori error estimates corresponding to the second approach (continuous and piecewise linear multipliers). Our second purpose is to implement numerically both approaches and to compare them to several significant examples.

The paper is organized as follows. First, we introduce the equations modelling the frictionless unilateral contact problem between elastic bodies. Then we establish the continuous mixed variational formulation of the problem in section 2 .

In the third section, we propose different well-posed finite element approaches in order to approximate the mixed problem. The following section is devoted to the a priori error estimations committed by the mixed finite element approximations. In particular, we show an optimal error estimate of order $h$ for finite elements of degree one.

Received by the editor November 5, 1999 and, in revised form, March 14, 2000.

2000 Mathematics Subject Classification. Primary 65N30, 74M15.

Key words and phrases. Mixed finite element methods, unilateral contact problems, a priori error estimates. 
Finally the algebraic formulations of the problem are given and the corresponding numerical experiments are achieved in the fifth section.

We consider two bodies which occupy, in the initial stage without stress, two bounded domains $\Omega^{1}$ and $\Omega^{2}$ of the two-dimensional space. For $\ell=1,2$, the boundary $\partial \Omega^{\ell}$ of $\Omega^{\ell}$ is assumed to be "smooth" and is the union of three nonoverlapping portions $\Gamma_{u}^{\ell}, \Gamma_{g}^{\ell}$ and $\Gamma_{c}^{\ell}$. Both domains $\Omega^{1}$ and $\Omega^{2}$ have a common contact part denoted $\Gamma_{c}=\Gamma_{c}^{1}=\Gamma_{c}^{2}$. The normal unit outward vector on $\partial \Omega^{\ell}$ is denoted $\boldsymbol{n}^{\ell}=\left(n_{1}^{\ell}, n_{2}^{\ell}\right)$. Both bodies are subjected to volume forces $\boldsymbol{f}^{\ell}=\left(f_{1}^{\ell}, f_{2}^{\ell}\right)$ and surface forces $\boldsymbol{g}^{\ell}=\left(g_{1}^{\ell}, g_{2}^{\ell}\right)$ are applied to the boundary part $\Gamma_{g}^{\ell}$.

The frictionless unilateral contact problem consists of finding the displacement fields $\boldsymbol{u}=\left(\boldsymbol{u}^{1}, \boldsymbol{u}^{2}\right)$ (where the notation $\boldsymbol{u}^{\ell}$ stands for $\left.\left.\boldsymbol{u}\right|_{\Omega^{\ell}}\right)$ with $\boldsymbol{u}^{\ell}=\left(u_{1}^{\ell}, u_{2}^{\ell}\right), 1 \leq$ $\ell \leq 2$, which satisfy the equations and conditions 1.1 1.10 for $\ell=1,2$ :

$$
\frac{\partial \sigma_{i j}^{\ell}}{\partial x_{j}}+f_{i}^{\ell}=0 \quad \text { in } \Omega^{\ell}
$$

this is the equilibrium equation where the summation convention of repeated indices is adopted and where $\sigma^{\ell}=\left(\sigma_{i j}^{\ell}\right), 1 \leq i, j \leq 2$, denotes the stress tensor field linked to the displacements by the constitutive relation

$$
\sigma_{i j}^{\ell}=a_{i j k h}^{\ell} \varepsilon_{k h}^{\ell}\left(\boldsymbol{u}^{\ell}\right) \quad \text { in } \Omega^{\ell}
$$

with

$$
a_{i j k h}^{\ell}=a_{j i k h}^{\ell}=a_{k h i j}^{\ell}, \quad \varepsilon_{i j}^{\ell}=\frac{1}{2}\left(\frac{\partial u_{i}^{\ell}}{\partial x_{j}}+\frac{\partial u_{j}^{\ell}}{\partial x_{i}}\right) .
$$

The notation $\varepsilon^{\ell}$ represents the linearized strain tensor field. The equations on the boundary parts restricted to Dirichlet and Neumann conditions are:

$$
\begin{aligned}
u_{i}^{\ell} & =0 & & \text { on } \Gamma_{u}^{\ell}, \\
\sigma_{i j}^{\ell} n_{j}^{\ell} & =g_{i}^{\ell} & & \text { on } \Gamma_{g}^{\ell} .
\end{aligned}
$$

The conditions on the boundary part $\Gamma_{c}$ constrained by frictionless unilateral contact conditions incorporate the Signorini conditions:

$$
\begin{aligned}
u_{i}^{1} n_{i}^{1}+u_{i}^{2} n_{i}^{2} & \leq 0 \\
\sigma_{i j}^{1} n_{j}^{1} n_{i}^{1}=\sigma_{i j}^{2} n_{j}^{2} n_{i}^{2} & \leq 0 \\
\sigma_{i j}^{\ell} n_{j}^{\ell} n_{i}^{\ell}<0 & \Rightarrow u_{i}^{1} n_{i}^{1}+u_{i}^{2} n_{i}^{2}=0, \\
u_{i}^{1} n_{i}^{1}+u_{i}^{2} n_{i}^{2}<0 & \Rightarrow \sigma_{i j}^{\ell} n_{j}^{\ell} n_{i}^{\ell}=0 .
\end{aligned}
$$

The notation $u_{i}^{1} n_{i}^{1}+u_{i}^{2} n_{i}^{2}$ represents the jump of the normal relative displacement across the contact zone $\Gamma_{c}$ : either contact (i.e., $u_{i}^{1} n_{i}^{1}+u_{i}^{2} n_{i}^{2}=0$ ) or separation (i.e., $u_{i}^{1} n_{i}^{1}+u_{i}^{2} n_{i}^{2}<0$ ) are allowed. In other words (1.6) is the nonpenetration condition. The terms $\sigma_{i j}^{\ell} n_{j}^{\ell} n_{i}^{\ell}, \ell=1,2$, are the normal components of $\sigma_{i j}^{\ell} n_{j}^{\ell}$ (also called normal constraints) which are equal according to the action and the reaction principle and nonpositive. Finally (1.8)-(1.9) are the complementarity conditions.

The conditions expressing the absence of friction are as follows:

$$
\sigma_{k j}^{\ell} n_{j}^{\ell}=\left(\sigma_{i j}^{\ell} n_{j}^{\ell} n_{i}^{\ell}\right) n_{k}^{\ell}
$$

this means that for $\ell=1,2$ the tangential component of $\sigma_{i j}^{\ell} n_{j}^{\ell}$ is equal to zero. 


\section{VARIATIONAL FORMULATION}

We begin with some notation used hereafter. Let $\Omega \subset \mathbb{R}^{2}$ be a Lipschitz domain whose generic point is denoted $\boldsymbol{x}=\left(x_{1}, x_{2}\right)$. The Lebesgue space $L^{2}(\Omega)$ is endowed with the inner product

$$
(\varphi, \psi)=\int_{\Omega} \varphi \psi d \boldsymbol{x}
$$

and the standard Sobolev spaces $H^{m}(\Omega), m \geq 1$, are equipped with the norm

$$
\|\psi\|_{H^{m}(\Omega)}=\left(\sum_{0 \leq|\alpha| \leq m}\left\|D^{\alpha} \psi\right\|_{L^{2}(\Omega)}^{2}\right)^{\frac{1}{2}},
$$

where $\alpha=\left(\alpha_{1}, \alpha_{2}\right)$ is a multi-index in $\mathbb{N}^{2}$ and the symbol $D^{\alpha}$ denotes a partial derivative. The convention $H^{0}(\Omega)=L^{2}(\Omega)$ is adopted. In order to obtain the mixed variational formulation corresponding to the problem (1.1)-(1.10), we introduce the Hilbert space $\left(H^{1}\left(\Omega^{1}\right)\right)^{2} \times\left(H^{1}\left(\Omega^{2}\right)\right)^{2}$ endowed with the norm

$$
\|\cdot\|=\left(\sum_{\ell=1}^{2}\|\cdot\|_{\left(H^{1}\left(\Omega^{\ell}\right)\right)^{2}}^{2}\right)^{\frac{1}{2}}
$$

and the subspace $\mathbf{V}=\mathbf{V}\left(\Omega^{1}\right) \times \mathbf{V}\left(\Omega^{2}\right)$ where

$$
\mathbf{V}\left(\Omega^{\ell}\right)=\left\{\boldsymbol{v}^{\ell} \in\left(H^{1}\left(\Omega^{\ell}\right)\right)^{2}, \quad \boldsymbol{v}^{\ell}=0 \text { on } \Gamma_{u}^{\ell}\right\} .
$$

Next, we define the convex cone of Lagrange multipliers denoted $M$ and defined as follows:

$$
M=\left\{\mu \in H^{-\frac{1}{2}}\left(\Gamma_{c}\right), \quad\langle\mu, \psi\rangle_{-\frac{1}{2}, \frac{1}{2}} \geq 0 \text { for all } \psi \in H^{\frac{1}{2}}\left(\Gamma_{c}\right), \psi \geq 0\right\},
$$

where $\langle., .\rangle_{-\frac{1}{2}, \frac{1}{2}}$ denotes the duality pairing between $H^{\frac{1}{2}}\left(\Gamma_{c}\right)$ and its topological dual space $H^{-\frac{1}{2}}\left(\Gamma_{c}\right)$. The norm on $H^{\tau}\left(\Gamma_{c}\right), \tau \in \mathbb{R}_{+} \backslash \mathbb{N}$, is given by (see [1], Theorem 7.48)

$$
\|\psi\|_{H^{\tau}\left(\Gamma_{c}\right)}=\left(\|\psi\|_{H^{m}\left(\Gamma_{c}\right)}^{2}+\int_{\Gamma_{c}} \int_{\Gamma_{c}} \frac{\left(D^{m} \psi(\boldsymbol{x})-D^{m} \psi(\boldsymbol{y})\right)^{2}}{|\boldsymbol{x}-\boldsymbol{y}|^{1+2 \theta}} d \Gamma d \Gamma\right)^{\frac{1}{2}},
$$

where $m$ is the integer part of $\tau$ and $\theta$ its decimal part. In the previous integral, $D^{m} \psi$ stands for the $m$-order derivative of $\psi$ along $\Gamma_{c}$ and $d \Gamma$ denotes the linear measure on $\Gamma_{c}$. The dual norm $\|\cdot\|_{H^{-\frac{1}{2}}\left(\Gamma_{c}\right)}$ on $H^{-\frac{1}{2}}\left(\Gamma_{c}\right)$ is

$$
\|\psi\|_{H^{-\frac{1}{2}}\left(\Gamma_{c}\right)}=\sup _{\varphi \in H^{\frac{1}{2}}\left(\Gamma_{c}\right)} \frac{\langle\psi, \varphi\rangle_{-\frac{1}{2}, \frac{1}{2}}}{\|\varphi\|_{H^{\frac{1}{2}}\left(\Gamma_{c}\right)}} .
$$

We will also make use of the following norms:

$$
\|\psi\|_{L^{\infty}\left(\Gamma_{c}\right)}=\operatorname{ess} \sup _{x \in \Gamma_{c}}|\psi(x)| \quad \text { and } \quad\|\psi\|_{W^{1, \infty}\left(\Gamma_{c}\right)}=\max _{\alpha=0,1}\left\|D^{\alpha} \psi(\boldsymbol{x})\right\|_{L^{\infty}\left(\Gamma_{c}\right)} .
$$


Let $\boldsymbol{u}=\left(\boldsymbol{u}^{1}, \boldsymbol{u}^{2}\right)$ and $\boldsymbol{v}=\left(\boldsymbol{v}^{1}, \boldsymbol{v}^{2}\right)$ in $\mathbf{V}$ and $\mu$ in $H^{-\frac{1}{2}}\left(\Gamma_{c}\right)$. We define

$$
\begin{aligned}
& a(\boldsymbol{u}, \boldsymbol{v})=\sum_{\ell=1}^{2} \int_{\Omega^{\ell}} a_{i j k h}^{\ell} \varepsilon_{i j}^{\ell}\left(\boldsymbol{u}^{\ell}\right) \varepsilon_{k h}^{\ell}\left(\boldsymbol{v}^{\ell}\right) d \Omega^{\ell}, \\
& L(\boldsymbol{v})=\sum_{\ell=1}^{2}\left(\int_{\Omega^{\ell}} f_{i}^{\ell} v_{i}^{\ell} d \Omega^{\ell}+\int_{\Gamma_{g}^{\ell}} g_{i}^{\ell} v_{i}^{\ell} d \Gamma^{\ell}\right),
\end{aligned}
$$

and, denoting $\boldsymbol{v}^{\ell}=\left(v_{1}^{\ell}, v_{2}^{\ell}\right), \ell=1,2$,

$$
b(\mu, \boldsymbol{v})=\left\langle\mu, v_{i}^{1} n_{i}^{1}+v_{i}^{2} n_{i}^{2}\right\rangle_{-\frac{1}{2}, \frac{1}{2}}=\left\langle\mu, \boldsymbol{v}^{1} \cdot \boldsymbol{n}^{1}+\boldsymbol{v}^{2} \cdot \boldsymbol{n}^{2}\right\rangle_{-\frac{1}{2}, \frac{1}{2}},
$$

where the notation - obviously denotes the inner product of $\mathbb{R}^{2}$.

We consider that $\left(f_{1}^{\ell}, f_{2}^{\ell}\right) \in\left(L^{2}\left(\Omega^{\ell}\right)\right)^{2},\left(g_{1}^{\ell}, g_{2}^{\ell}\right) \in\left(L^{2}\left(\Gamma_{g}^{\ell}\right)\right)^{2},\left(a_{i j k h}^{\ell}\right)_{1 \leq i, j, k, h \leq 2} \in$ $\left(L^{\infty}\left(\Omega^{\ell}\right)\right)^{16}$. We suppose that $\alpha^{\ell}$ exists verifying $a_{i j, k h}^{\ell} \tau_{i j} \tau_{k h} \geq \alpha^{\ell} \tau_{i j} \tau_{i j}$ for all $\tau_{i j}=\tau_{j i}$. Moreover we assume that the surface measure of $\Gamma_{u}^{\ell}$ does not vanish. Notice that the latter hypothesis could be avoided in the forthcoming study, if for example the loads are well-oriented (see [1]).

The mixed formulation of the unilateral contact problem without friction consists then of finding $\boldsymbol{u} \in \mathbf{V}$ and $\lambda \in M$ so that

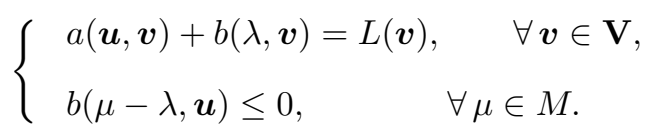

The existence and uniqueness statement for this saddle-point problem has been established in 11, chapter III, Theorem 9.4. We recall this result in the following proposition.

Proposition 2.1. Problem (2.1) admits a unique solution $(\boldsymbol{u}, \lambda) \in \mathbf{V} \times M$. We have

$$
\lambda=-\sigma_{i j}^{1} n_{j}^{1} n_{i}^{1}=-\sigma_{i j}^{2} n_{j}^{2} n_{i}^{2} .
$$

Remark 2.2. Let $(\boldsymbol{u}, \lambda)$ be the solution of problem (2.1).

Then $(\boldsymbol{u}, \lambda)$ is the saddle-point of the functional $\mathcal{L}(.,$.$) over \mathbf{V} \times M$ where

$$
\mathcal{L}(\boldsymbol{v}, \mu)=\frac{1}{2} a(\boldsymbol{v}, \boldsymbol{v})+b(\mu, \boldsymbol{v})-L(\boldsymbol{v}) .
$$

Moreover $\boldsymbol{u}$ is the solution of the variational inequality (see [9, 14, 15])

$$
\boldsymbol{u} \in \mathbf{K}, \quad a(\boldsymbol{u}, \boldsymbol{v}-\boldsymbol{u}) \geq L(\boldsymbol{v}-\boldsymbol{u}), \quad \forall \boldsymbol{v} \in \mathbf{K},
$$

and also of the minimization problem

$$
\boldsymbol{u} \in \mathbf{K}, \quad J(\boldsymbol{u})=\min _{\boldsymbol{v} \in \mathbf{K}} J(\boldsymbol{v}),
$$

where

$$
\mathbf{K}=\left\{\boldsymbol{v}=\left(\boldsymbol{v}^{1}, \boldsymbol{v}^{2}\right) \in \mathbf{V}, \quad \boldsymbol{v}^{1} \cdot \boldsymbol{n}^{1}+\boldsymbol{v}^{2} \cdot \boldsymbol{n}^{2} \leq 0 \text { on } \Gamma_{c}\right\},
$$

and

$$
J(\boldsymbol{v})=\frac{1}{2} a(\boldsymbol{v}, \boldsymbol{v})-L(\boldsymbol{v}) .
$$




\section{Finite ElEment approximation}

The present section is devoted to the finite element approximation of the saddlepoint problem (2.1). The key point lies in the finite element translation of the closed convex cone of Lagrange multipliers $M$. As a matter of fact, this type of approximation must lead not only to a well-posed discrete problem but also to good convergence rates of the discretized saddle-point problem towards the solution of (2.1).

Notice that we must distinguish the two independent problems arising in the building of the approximation model:

- the choice of the finite element space (approximating $H^{-\frac{1}{2}}\left(\Gamma_{c}\right)$ ) for the Lagrange multipliers,

- the choice of the approximated nonnegativity condition.

The first point is of more general concern whereas the second one is specific to inequality problems. Notice that both above-mentioned points also arise in a standard scalar valued Signorini problem. That means that these choices can be considered to be independent of matching or nonmatching meshes on the contact interface.

We suppose, for the sake of simplicity, that each subdomain $\Omega^{\ell}, \ell=1,2$, is a polygon and that $\Gamma_{c}$ is a straight line segment $\left[\boldsymbol{c}_{1}, \boldsymbol{c}_{2}\right]$ parallel to the $x_{2}$-axis. With each subdomain $\Omega^{\ell}$, we then associate a regular family of discretizations $\mathcal{T}_{h}^{\ell}$ made of triangular elements denoted $\kappa$ so that

$$
\overline{\Omega^{\ell}}=\bigcup_{\kappa \in \mathcal{T}_{h}^{\ell}} \bar{\kappa} .
$$

The discretization parameter $h_{\ell}$ on $\Omega^{\ell}$ is given by

$$
h_{\ell}=\max _{\kappa \in \mathcal{T}_{h}^{\ell}} h_{\kappa},
$$

where $h_{\kappa}$ denotes the diameter of the triangle $\kappa$. Let $h=\max \left(h_{1}, h_{2}\right)$. Denoting by $\rho_{\kappa}$ the diameter of the inscribed circle in $\kappa$, we assume that there exists a constant $c$ independent of the discretization parameter $h_{\ell}$ and satisfying

$$
\min _{\kappa \in \mathcal{T}_{h}^{\ell}} \frac{\rho_{\kappa}}{h_{\kappa}} \geq c .
$$

We suppose that the end points $\boldsymbol{c}_{1}$ and $\boldsymbol{c}_{2}$ of the contact zone $\Gamma_{c}$ are common nodes of the triangulations $\mathcal{T}_{h}^{1}$ and $\mathcal{T}_{h}^{2}$ and that the monodimensional traces of triangulations of $\mathcal{T}_{h}^{1}$ and $\mathcal{T}_{h}^{2}$ on $\Gamma_{c}$ are uniformly regular. For any integer $q \geq 0$, the notation $\mathbb{P}_{q}(\kappa)$ denotes the space of the polynomials with the global degree $\leq q$ on $\kappa$. The finite element space used in $\Omega^{\ell}$ is then defined by

$$
\mathbf{V}_{h}\left(\Omega^{\ell}\right)=\left\{\boldsymbol{v}_{h}^{\ell} \in\left(\mathcal{C}\left(\bar{\Omega}^{\ell}\right)\right)^{2}, \quad \forall \kappa \in \mathcal{T}_{h}^{\ell},\left.\quad \boldsymbol{v}_{h}^{\ell}\right|_{\kappa} \in\left(\mathbb{P}_{1}(\kappa)\right)^{2},\left.\quad \boldsymbol{v}_{h}^{\ell}\right|_{\Gamma_{u}^{\ell}}=0\right\},
$$

and the approximation space of $\mathbf{V}$ becomes

$$
\mathbf{V}_{h}=\mathbf{V}_{h}\left(\Omega^{1}\right) \times \mathbf{V}_{h}\left(\Omega^{2}\right) .
$$

The set of nodes on $\Gamma_{c}$ belonging to triangulation $\mathcal{T}_{h}^{\ell}$ are denoted

$$
\xi_{h}^{\ell}=\left\{\boldsymbol{c}_{1}=\boldsymbol{x}_{0}^{\ell}, \boldsymbol{x}_{1}^{\ell}, \ldots, \boldsymbol{x}_{N(h)-1}^{\ell}, \boldsymbol{x}_{N(h)}^{\ell}=\boldsymbol{c}_{2}\right\}
$$


such that the second component of $\boldsymbol{x}_{i}^{\ell}$ is increasing with $i$. In order to express the contact constraints by using conveniently chosen Lagrange multipliers on the contact zone, we have to introduce first the two spaces describing the degree of the polynomial approximation:

$$
\begin{aligned}
& W_{h}^{0, \ell}\left(\Gamma_{c}\right)=\left\{\mu_{h},\left.\mu_{h}\right|_{]} \boldsymbol{x}_{i}^{\ell}, \boldsymbol{x}_{i+1}^{\ell}\left[\in \mathbb{P}_{0}(] \boldsymbol{x}_{i}^{\ell}, \boldsymbol{x}_{i+1}^{\ell}[), 0 \leq i \leq N(h)-1\right\}\right. \\
& W_{h}^{1, \ell}\left(\Gamma_{c}\right)=\left\{\mu_{h} \in \mathcal{C}\left(\overline{\Gamma_{c}}\right), \exists \boldsymbol{v}_{h}^{\ell} \in \mathbf{V}_{h}\left(\Omega^{\ell}\right) \text { such that } \boldsymbol{v}_{h}^{\ell} \cdot \boldsymbol{n}^{\ell}=\mu_{h} \text { on } \Gamma_{c}\right\} .
\end{aligned}
$$

The next step consists of choosing one of the previous spaces and then trying to approximate the nonnegativity condition incorporated in the definition of $M$. We first consider the space $W_{h}^{0, \ell}\left(\Gamma_{c}\right)$ which leads to the following natural definition:

$$
M_{h}^{0, \ell}=\left\{\mu_{h} \in W_{h}^{0, \ell}\left(\Gamma_{c}\right), \mu_{h} \geq 0 \text { a.e. on } \Gamma_{c}\right\} \text {. }
$$

The choice of the space $W_{h}^{1, \ell}\left(\Gamma_{c}\right)$ allows us to define successively the two following approximation convex cones:

$$
M_{h}^{1, \ell}=\left\{\mu_{h} \in W_{h}^{1, \ell}\left(\Gamma_{c}\right), \mu_{h} \geq 0 \text { on } \Gamma_{c}\right\}
$$

and

$$
M_{h}^{1, \ell, *}=\left\{\mu_{h} \in W_{h}^{1, \ell}\left(\Gamma_{c}\right), \int_{\Gamma_{c}} \mu_{h} \psi_{h} d \Gamma \geq 0, \forall \psi_{h} \in M_{h}^{1, \ell}\right\}
$$

Remark 3.1. One obtains $M_{h}^{0, \ell} \subset M, M_{h}^{1, \ell} \subset M$ and $M_{h}^{1, \ell} \subset M_{h}^{1, \ell, *}$. Moreover, it is easy to check that $M_{h}^{1, \ell, *} \not \subset M$.

The discretized mixed formulations of the unilateral contact problem without friction consist then of finding $\boldsymbol{u}_{h} \in \mathbf{V}_{h}$ and $\lambda_{h} \in M_{h}$ satisfying

$$
\left\{\begin{array}{l}
a\left(\boldsymbol{u}_{h}, \boldsymbol{v}_{h}\right)+b\left(\lambda_{h}, \boldsymbol{v}_{h}\right)=L\left(\boldsymbol{v}_{h}\right), \quad \forall \boldsymbol{v}_{h} \in \mathbf{V}_{h}, \\
b\left(\mu_{h}-\lambda_{h}, \boldsymbol{u}_{h}\right) \leq 0, \quad \forall \mu_{h} \in M_{h},
\end{array}\right.
$$

where $M_{h}=M_{h}^{0, \ell}$ or $M_{h}=M_{h}^{1, \ell}$ or $M_{h}=M_{h}^{1, \ell, *}$ with $\ell=1$ or 2 .

The first studies and convergence results corresponding to $M_{h}=M_{h}^{0, \ell}$ (in the case of a body in contact with a rigid foundation) have been made in [11] and some recent improvements and extensions can be found in [17]. Now we shall focus on the other cases: $M_{h}=M_{h}^{1, \ell}$ or $M_{h}=M_{h}^{1, \ell, *}$.

The $\mathbf{V}_{h}$-ellipticity of the bilinear symmetrical form $a(.,$.$) follows from standard$ results (see for example [4]). Therefore, the first argument $\boldsymbol{u}_{h}$ solution to the problem (3.4) is unique. In order to prove the existence and the uniqueness of the saddle-point of (3.4), it is only necessary to verify that

$$
\left\{\mu_{h} \in M_{h}, \quad b\left(\mu_{h}, \boldsymbol{v}_{h}\right)=0, \forall \boldsymbol{v}_{h} \in \mathbf{V}_{h}\right\}=\{0\},
$$

which is obvious. As a consequence, we obtain the following statement:

Proposition 3.2. Let $M_{h}=M_{h}^{1, \ell}$ or $M_{h}=M_{h}^{1, \ell, *}$ with $\ell=1$ or 2 . Then problem (3.4) admits a unique solution $\left(\boldsymbol{u}_{h}, \lambda_{h}\right) \in \mathbf{V}_{h} \times M_{h}$.

In the forthcoming convergence analysis, we will need more information about the compatibility between the spaces $W_{h}^{1, \ell}\left(\Gamma_{c}\right)$ and $\mathbf{V}_{h}$ so that we will have to consider the corresponding inf-sup condition. This condition is given by the following 
proposition in which we suppose that the neighboring boundary parts of the contact zone are restricted to Neumann type conditions (1.5). Note that the latter assumption is not restrictive and becomes quite natural in engineering applications.

Proposition 3.3. Suppose that $\overline{\Gamma_{c}} \cap \overline{\Gamma_{u}^{\ell}}=\emptyset$ for $\ell=1,2$. Then the following inf-sup condition holds:

$$
\inf _{\mu_{h} \in W_{h}^{1, \ell}\left(\Gamma_{c}\right)} \sup _{\boldsymbol{v}_{h} \in \mathbf{V}_{h}} \frac{b\left(\mu_{h}, \boldsymbol{v}_{h}\right)}{\left\|\mu_{h}\right\|_{H^{-\frac{1}{2}}\left(\Gamma_{c}\right)}\left\|\boldsymbol{v}_{h}\right\|} \geq \beta>0,
$$

where $\beta$ is independent of $h$.

Proof. We introduce the projection operator $\pi_{h}^{\ell}$ on $W_{h}^{1, \ell}\left(\Gamma_{c}\right)$ defined for any function $\varphi \in L^{2}\left(\Gamma_{c}\right)$ by

$$
\pi_{h}^{\ell} \varphi \in W_{h}^{1, \ell}\left(\Gamma_{c}\right), \quad \int_{\Gamma_{c}}\left(\pi_{h}^{\ell} \varphi-\varphi\right) \mu_{h}^{\ell} d \Gamma=0, \quad \forall \mu_{h}^{\ell} \in W_{h}^{1, \ell}\left(\Gamma_{c}\right) .
$$

By definition, the operator $\pi_{h}^{\ell}$ is stable in the $L^{2}\left(\Gamma_{c}\right)$-norm.

Let $\varphi \in H^{1}\left(\Gamma_{c}\right)$; then

$$
\left\|\pi_{h}^{\ell} \varphi\right\|_{H^{1}\left(\Gamma_{c}\right)} \leq\left\|\pi_{h}^{\ell}\left(\varphi-i_{h}^{\ell} \varphi\right)\right\|_{H^{1}\left(\Gamma_{c}\right)}+\left\|i_{h}^{\ell} \varphi\right\|_{H^{1}\left(\Gamma_{c}\right)},
$$

where $i_{h}^{\ell}$ denotes the Lagrange interpolation operator ranging in $W_{h}^{1, \ell}\left(\Gamma_{c}\right)$. Of course, we have $\pi_{h}^{\ell}\left(i_{h}^{\ell} \varphi\right)=i_{h}^{\ell} \varphi$ owing to the assumption $\overline{\Gamma_{c}} \cap \overline{\Gamma_{u}^{\ell}}=\emptyset$. Let us denote, by $\tilde{h}_{\ell}$, the largest length of the $1 \mathrm{D}$-meshes of $\Omega^{\ell}$ on $\Gamma_{c}$. The uniform regularity of these $1 \mathrm{D}$-meshes on $\Gamma_{c}$ allows the use of the inverse inequality that leads to

$$
\begin{aligned}
\left\|\pi_{h}^{\ell}\left(\varphi-i_{h}^{\ell} \varphi\right)\right\|_{H^{1}\left(\Gamma_{c}\right)} \leq C \tilde{h}_{\ell}^{-1}\left\|\pi_{h}^{\ell}\left(\varphi-i_{h}^{\ell} \varphi\right)\right\|_{L^{2}\left(\Gamma_{c}\right)} & \leq C \tilde{h}_{\ell}^{-1}\left\|\varphi-i_{h}^{\ell} \varphi\right\|_{L^{2}\left(\Gamma_{c}\right)} \\
& \leq C_{1}\|\varphi\|_{H^{1}\left(\Gamma_{c}\right)} .
\end{aligned}
$$

Moreover it can be easily shown that the operator $i_{h}^{\ell}$ is stable in the $H^{1}\left(\Gamma_{c}\right)$-norm. Consequently the operator $\pi_{h}^{\ell}$ is stable in the $H^{1}\left(\Gamma_{c}\right)$-norm. So we conclude that a positive constant $C$ independent of $h$ exists so that

$$
\left\|\pi_{h}^{\ell} \varphi\right\|_{H^{\frac{1}{2}}\left(\Gamma_{c}\right)} \leq C\|\varphi\|_{H^{\frac{1}{2}}\left(\Gamma_{c}\right)}, \quad \forall \varphi \in H^{\frac{1}{2}}\left(\Gamma_{c}\right),
$$

by using an Hilbertian interpolation argument of index $1 / 2$ between $L^{2}\left(\Gamma_{c}\right)$ and $H^{1}\left(\Gamma_{c}\right)$. Next, we show that inequality (3.5) is a consequence of (3.7).

Let $\mu_{h}$ belong to $W_{h}^{1, \ell}\left(\Gamma_{c}\right)$. There is $\psi \in H^{\frac{1}{2}}\left(\Gamma_{c}\right)$ with $\|\psi\|_{H^{\frac{1}{2}}\left(\Gamma_{c}\right)}=1$, so that

$$
\left\langle\mu_{h}, \psi\right\rangle_{-\frac{1}{2}, \frac{1}{2}}=\int_{\Gamma_{c}} \mu_{h} \psi d \Gamma=\left\|\mu_{h}\right\|_{H^{-\frac{1}{2}}\left(\Gamma_{c}\right)} .
$$

The definition of $\pi_{h}^{\ell}$ in (3.6) leads to

$$
\int_{\Gamma_{c}} \mu_{h} \psi d \Gamma=\int_{\Gamma_{c}} \mu_{h} \pi_{h}^{\ell} \psi d \Gamma .
$$

We then consider an extension operator $R_{h}^{\ell}$ from $W_{h}^{1, \ell}\left(\Gamma_{c}\right)$ into $\mathbf{V}_{h}\left(\Omega^{\ell}\right)$ satisfying (see [16])

$$
R_{h}^{\ell}\left(\psi_{h}^{\ell}\right)=\psi_{h}^{\ell} \boldsymbol{n}^{\ell} \text { on } \Gamma_{c} \quad \text { and } \quad\left\|R_{h}^{\ell}\left(\psi_{h}^{\ell}\right)\right\|_{\left(H^{1}\left(\Omega^{\ell}\right)\right)^{2}} \leq C_{2}\left\|\psi_{h}^{\ell}\right\|_{H^{\frac{1}{2}}\left(\Gamma_{c}\right)} .
$$

So by setting

$$
\boldsymbol{w}_{h}^{\ell}=R_{h}^{\ell}\left(\pi_{h}^{\ell} \psi\right) \in \mathbf{V}_{h}\left(\Omega^{\ell}\right) \quad \text { and }\left.\quad \boldsymbol{w}_{h}\right|_{\Omega^{\ell}}=\boldsymbol{w}_{h}^{\ell},\left.\boldsymbol{w}_{h}\right|_{\Omega^{3-\ell}}=0,
$$


we obtain

$$
\left\|\boldsymbol{w}_{h}\right\| \leq C_{2}\left\|\pi_{h}^{\ell} \psi\right\|_{H^{\frac{1}{2}\left(\Gamma_{c}\right)}} \leq C_{3}\|\psi\|_{H^{\frac{1}{2}}\left(\Gamma_{c}\right)} .
$$

Taking into account that $\|\psi\|_{H^{\frac{1}{2}}\left(\Gamma_{c}\right)}=1$, we finally come to the conclusion that there is a positive constant $C$ independent of $h$ (and of $\mu_{h}$ ) such that

$$
\begin{aligned}
& C\left\|\mu_{h}\right\|_{H^{-\frac{1}{2}}\left(\Gamma_{c}\right)} \leq \frac{\left\|\mu_{h}\right\|_{H^{-\frac{1}{2}}\left(\Gamma_{c}\right)}}{\left\|\boldsymbol{w}_{h}\right\|}=\frac{\int_{\Gamma_{c}} \mu_{h}\left(\boldsymbol{w}_{h}^{\ell} \cdot \boldsymbol{n}^{\ell}\right) d \Gamma}{\left\|\boldsymbol{w}_{h}\right\|}=\frac{b\left(\mu_{h}, \boldsymbol{w}_{h}\right)}{\left\|\boldsymbol{w}_{h}\right\|} \\
& \leq \sup _{\boldsymbol{v}_{h} \in \mathbf{V}_{h}} \frac{b\left(\mu_{h}, \boldsymbol{v}_{h}\right)}{\left\|\boldsymbol{v}_{h}\right\|}
\end{aligned}
$$

Then the inf-sup condition (3.5) is proved to be true.

\section{ERROR ANALYSIS}

This section consists of obtaining a priori error estimates corresponding to the mixed finite element approximations. The starting point is the following lemma.

Lemma 4.1. Let $(\boldsymbol{u}, \lambda)$ be the solution of 2.1 and let $\left(\boldsymbol{u}_{h}, \lambda_{h}\right)$ be the solution of (3.4). Then for any $\boldsymbol{v}_{h} \in \mathbf{V}_{h}$ and $\mu_{h} \in M_{h}$ we obtain

$$
\begin{aligned}
a\left(\boldsymbol{u}-\boldsymbol{u}_{h}, \boldsymbol{u}-\boldsymbol{u}_{h}\right) \leq & a\left(\boldsymbol{u}-\boldsymbol{u}_{h}, \boldsymbol{u}-\boldsymbol{v}_{h}\right)+b\left(\lambda-\mu_{h}, \boldsymbol{u}_{h}-\boldsymbol{u}\right)+b\left(\lambda-\lambda_{h}, \boldsymbol{u}-\boldsymbol{v}_{h}\right) \\
& +b\left(\lambda-\mu_{h}, \boldsymbol{u}\right)+b\left(\lambda_{h}, \boldsymbol{u}\right),
\end{aligned}
$$

where $M_{h}=M_{h}^{1, \ell}$ or $M_{h}=M_{h}^{1, \ell, *}$ with $\ell=1$ or 2 .

Proof. Let $\boldsymbol{v}_{h}$ be an element of $\mathbf{V}_{h}$. It follows that

$$
a\left(\boldsymbol{u}-\boldsymbol{u}_{h}, \boldsymbol{u}-\boldsymbol{u}_{h}\right)=a\left(\boldsymbol{u}-\boldsymbol{u}_{h}, \boldsymbol{u}-\boldsymbol{v}_{h}\right)+a\left(\boldsymbol{u}-\boldsymbol{u}_{h}, \boldsymbol{v}_{h}-\boldsymbol{u}_{h}\right) .
$$

Using the first equations of problems 2.1 and 3.4 this gives

$$
\begin{aligned}
a\left(\boldsymbol{u}-\boldsymbol{u}_{h}, \boldsymbol{v}_{h}-\boldsymbol{u}_{h}\right) & =L\left(\boldsymbol{v}_{h}-\boldsymbol{u}_{h}\right)-b\left(\lambda, \boldsymbol{v}_{h}-\boldsymbol{u}_{h}\right)-L\left(\boldsymbol{v}_{h}-\boldsymbol{u}_{h}\right)+b\left(\lambda_{h}, \boldsymbol{v}_{h}-\boldsymbol{u}_{h}\right) \\
& =b\left(\lambda, \boldsymbol{u}_{h}-\boldsymbol{v}_{h}\right)+b\left(\lambda_{h}, \boldsymbol{v}_{h}-\boldsymbol{u}_{h}\right) .
\end{aligned}
$$

Noticing that $b\left(\lambda_{h}, \boldsymbol{u}_{h}\right)=0$, we deduce that

$$
a\left(\boldsymbol{u}-\boldsymbol{u}_{h}, \boldsymbol{u}-\boldsymbol{u}_{h}\right)=a\left(\boldsymbol{u}-\boldsymbol{u}_{h}, \boldsymbol{u}-\boldsymbol{v}_{h}\right)+b\left(\lambda, \boldsymbol{u}_{h}-\boldsymbol{v}_{h}\right)+b\left(\lambda_{h}, \boldsymbol{v}_{h}\right) .
$$

For any $\mu_{h} \in M_{h}$, we obtain

$$
\begin{aligned}
a\left(\boldsymbol{u}-\boldsymbol{u}_{h}, \boldsymbol{u}-\boldsymbol{u}_{h}\right)= & a\left(\boldsymbol{u}-\boldsymbol{u}_{h}, \boldsymbol{u}-\boldsymbol{v}_{h}\right)+b\left(\mu_{h}-\lambda, \boldsymbol{u}-\boldsymbol{u}_{h}\right)+b\left(\lambda-\lambda_{h}, \boldsymbol{u}-\boldsymbol{v}_{h}\right) \\
& +b\left(\lambda-\mu_{h}, \boldsymbol{u}\right)+b\left(\lambda_{h}, \boldsymbol{u}\right)+b\left(\mu_{h}, \boldsymbol{u}_{h}\right) .
\end{aligned}
$$

The inequality of (3.4) implies that $b\left(\mu_{h}, \boldsymbol{u}_{h}\right) \leq 0$ for any $\mu_{h} \in M_{h}$. This ends the proof of the lemma.

We now attempt to derive an upper bound of the terms involved in the previous lemma.

Lemma 4.2. Let $(\boldsymbol{u}, \lambda)$ be the solution of (2.1). Suppose that $\boldsymbol{u}^{1} \in\left(H^{2}\left(\Omega^{1}\right)\right)^{2}$ and $\boldsymbol{u}^{2} \in\left(H^{2}\left(\Omega^{2}\right)\right)^{2}$. Let $\left(\boldsymbol{u}_{h}, \lambda_{h}\right)$ be the solution of (3.4) with $M_{h}=M_{h}^{1, \ell, *}$ and $\ell=1$ or 2 . Then we have the following estimate:

$$
\left\|\boldsymbol{u}-\boldsymbol{u}_{h}\right\|^{2} \leq C(\boldsymbol{u})\left(h\left\|\lambda-\lambda_{h}\right\|_{H^{-\frac{1}{2}}\left(\Gamma_{c}\right)}+C(\boldsymbol{u}) h^{\frac{3}{2}}\right),
$$

where the positive constant $C(\boldsymbol{u})$ depends linearly on $\left\|\boldsymbol{u}^{1}\right\|_{\left(H^{2}\left(\Omega^{1}\right)\right)^{2}}$ and $\left\|\boldsymbol{u}^{2}\right\|_{\left(H^{2}\left(\Omega^{2}\right)\right)^{2}}$. 
Proof. Using Lemma 4.1, we will show that $\boldsymbol{v}_{h} \in \mathbf{V}_{h}$ and $\mu_{h} \in M_{h}$ exist, satisfying

$$
\left\{\begin{aligned}
a\left(\boldsymbol{u}-\boldsymbol{u}_{h}, \boldsymbol{u}-\boldsymbol{v}_{h}\right) & \leq C(\boldsymbol{u}) h\left\|\boldsymbol{u}-\boldsymbol{u}_{h}\right\|, \\
b\left(\lambda-\mu_{h}, \boldsymbol{u}_{h}-\boldsymbol{u}\right) & \leq C(\boldsymbol{u}) h\left\|\boldsymbol{u}-\boldsymbol{u}_{h}\right\|, \\
b\left(\lambda-\lambda_{h}, \boldsymbol{u}-\boldsymbol{v}_{h}\right) & \leq C(\boldsymbol{u}) h\left\|\lambda-\lambda_{h}\right\|_{H^{-\frac{1}{2}}\left(\Gamma_{c}\right)}, \\
b\left(\lambda-\mu_{h}, \boldsymbol{u}\right) & \leq(C(\boldsymbol{u}))^{2} h^{2}, \\
b\left(\lambda_{h}, \boldsymbol{u}\right) & \leq C(\boldsymbol{u})\left(h\left\|\lambda-\lambda_{h}\right\|_{H^{-\frac{1}{2}}\left(\Gamma_{c}\right)}+C(\boldsymbol{u}) h^{\frac{3}{2}}\right) .
\end{aligned}\right.
$$

Before proving the above estimates, let us recall some approximation properties. Let $I_{h}^{\ell}$ and $i_{h}^{\ell}$ be the Lagrange interpolation operators with values respectively in $\mathbf{V}_{h}\left(\Omega^{\ell}\right)$ and $W_{h}^{1, \ell}\left(\Gamma_{c}\right)$. So, there is a constant $C>0$ satisfying for all $\boldsymbol{v}^{\ell} \in$ $\left(H^{2}\left(\Omega^{\ell}\right)\right)^{2}$ and $v \in H^{\frac{3}{2}}\left(\Gamma_{c}\right)$ (see [8])

$$
\left\|\boldsymbol{v}^{\ell}-I_{h}^{\ell} \boldsymbol{v}^{\ell}\right\| \leq C h_{\ell}\left\|\boldsymbol{v}^{\ell}\right\|_{\left(H^{2}\left(\Omega^{\ell}\right)\right)^{2}} \text { and }\left\|v-i_{h}^{\ell} v\right\|_{L^{2}\left(\Gamma_{c}\right)} \leq C h_{\ell}^{\frac{3}{2}}\|v\|_{H^{\frac{3}{2}}\left(\Gamma_{c}\right)} .
$$

Concerning the projection operator $\pi_{h}^{\ell}$ defined in (3.6), the following estimation holds (see [4]):

$$
\left\|\varphi-\pi_{h}^{\ell} \varphi\right\|_{H^{-\frac{1}{2}}\left(\Gamma_{c}\right)} \leq C h\|\varphi\|_{H^{\frac{1}{2}\left(\Gamma_{c}\right)}}, \quad \forall \varphi \in H^{\frac{1}{2}}\left(\Gamma_{c}\right) .
$$

Furthermore, if $\varphi \geq 0$ a.e. on $\Gamma_{c}$, then $\pi_{h}^{\ell} \varphi \in M_{h}^{1, \ell, *}$, because

$$
\int_{\Gamma_{c}}\left(\pi_{h}^{\ell} \varphi\right) \mu_{h} d \Gamma=\int_{\Gamma_{c}} \varphi \mu_{h} d \Gamma \geq 0, \quad \forall \mu_{h} \in M_{h}^{1, \ell} .
$$

Suppose henceforth that the elasticity coefficients are regular enough (e.g. $a_{i j k h}^{\ell} \in$ $\left.\mathcal{C}^{1}\left(\bar{\Omega}^{\ell}\right)\right)$ so that the trace theorem implies

$$
\|\lambda\|_{H^{\frac{1}{2}\left(\Gamma_{c}\right)}} \leq C\left\|\boldsymbol{u}^{\ell}\right\|_{\left(H^{2}\left(\Omega^{\ell}\right)\right)^{2}}, \quad \ell=1,2 .
$$

Now we choose $\boldsymbol{v}_{h}=I_{h} \boldsymbol{u}=\left(I_{h}^{1} \boldsymbol{u}^{1}, I_{h}^{2} \boldsymbol{u}^{2}\right)$ and $\mu_{h}=\pi_{h}^{\ell} \lambda$.

(i) The first term is evaluated by using the continuity of $a(.,$.$) and the property$ (4.1) which gives

$$
a\left(\boldsymbol{u}-\boldsymbol{u}_{h}, \boldsymbol{u}-\boldsymbol{v}_{h}\right) \leq C(\boldsymbol{u}) h\left\|\boldsymbol{u}-\boldsymbol{u}_{h}\right\| .
$$

(ii) This term is handled as follows with property (4.2):

$$
b\left(\lambda-\mu_{h}, \boldsymbol{u}_{h}-\boldsymbol{u}\right) \leq C\left\|\lambda-\pi_{h}^{\ell} \lambda\right\|_{H^{-\frac{1}{2}}\left(\Gamma_{c}\right)}\left\|\boldsymbol{u}_{h}-\boldsymbol{u}\right\| \leq C(\boldsymbol{u}) h\left\|\boldsymbol{u}_{h}-\boldsymbol{u}\right\| .
$$

(iii) Here again, we use estimate (4.1):

$$
b\left(\lambda-\lambda_{h}, \boldsymbol{u}-\boldsymbol{v}_{h}\right) \leq C\left\|\lambda-\lambda_{h}\right\|_{H^{-\frac{1}{2}\left(\Gamma_{c}\right)}}\left\|\boldsymbol{u}-\boldsymbol{v}_{h}\right\| \leq C(\boldsymbol{u}) h\left\|\lambda-\lambda_{h}\right\|_{H^{-\frac{1}{2}}\left(\Gamma_{c}\right)} .
$$

To evaluate this term, we invoke the definition of the $L^{2}$-projection operator:

$$
\begin{aligned}
b\left(\lambda-\mu_{h}, \boldsymbol{u}\right) & =\int_{\Gamma_{c}}\left(\lambda-\pi_{h}^{\ell} \lambda\right)\left(\boldsymbol{u}^{1} \cdot \boldsymbol{n}^{1}+\boldsymbol{u}^{2} \cdot \boldsymbol{n}^{2}\right) d \Gamma \\
& =\int_{\Gamma_{c}}\left(\lambda-\pi_{h}^{\ell} \lambda\right)\left(\boldsymbol{u}^{1} \cdot \boldsymbol{n}^{1}+\boldsymbol{u}^{2} \cdot \boldsymbol{n}^{2}-\pi_{h}^{\ell}\left(\boldsymbol{u}^{1} \cdot \boldsymbol{n}^{1}+\boldsymbol{u}^{2} \cdot \boldsymbol{n}^{2}\right)\right) d \Gamma \\
& \leq\left\|\lambda-\pi_{h}^{\ell} \lambda\right\|_{L^{2}\left(\Gamma_{c}\right)}\left\|\boldsymbol{u}^{1} \cdot \boldsymbol{n}^{1}+\boldsymbol{u}^{2} \cdot \boldsymbol{n}^{2}-\pi_{h}^{\ell}\left(\boldsymbol{u}^{1} \cdot \boldsymbol{n}^{1}+\boldsymbol{u}^{2} \cdot \boldsymbol{n}^{2}\right)\right\|_{L^{2}\left(\Gamma_{c}\right)} \\
& \leq(C(\boldsymbol{u}))^{2} h^{2} .
\end{aligned}
$$


(v) The last term leads to the following estimates:

$$
\begin{aligned}
b\left(\lambda_{h}, \boldsymbol{u}\right)= & \int_{\Gamma_{c}} \lambda_{h}\left(\boldsymbol{u}^{1} \cdot \boldsymbol{n}^{1}+\boldsymbol{u}^{2} \cdot \boldsymbol{n}^{2}\right) d \Gamma \\
= & \int_{\Gamma_{c}} \lambda_{h}\left(\boldsymbol{u}^{1} \cdot \boldsymbol{n}^{1}+\boldsymbol{u}^{2} \cdot \boldsymbol{n}^{2}-i_{h}^{\ell}\left(\boldsymbol{u}^{1} \cdot \boldsymbol{n}^{1}+\boldsymbol{u}^{2} \cdot \boldsymbol{n}^{2}\right)\right) d \Gamma \\
& \quad+\int_{\Gamma_{c}} \lambda_{h} i_{h}^{\ell}\left(\boldsymbol{u}^{1} \cdot \boldsymbol{n}^{1}+\boldsymbol{u}^{2} \cdot \boldsymbol{n}^{2}\right) d \Gamma .
\end{aligned}
$$

Noticing that $i_{h}^{\ell}\left(\boldsymbol{u}^{1} \cdot \boldsymbol{n}^{1}+\boldsymbol{u}^{2} \cdot \boldsymbol{n}^{2}\right) \leq 0, \lambda_{h} \in M_{h}^{1, \ell, *}$ and using the definition of $M_{h}^{1, \ell, *}$ allow us to write

$$
\int_{\Gamma_{c}} \lambda_{h} i_{h}^{\ell}\left(\boldsymbol{u}^{1} \cdot \boldsymbol{n}^{1}+\boldsymbol{u}^{2} \cdot \boldsymbol{n}^{2}\right) d \Gamma \leq 0
$$

Therefore

$$
\begin{aligned}
b\left(\lambda_{h}, \boldsymbol{u}\right) \leq & \int_{\Gamma_{c}} \lambda_{h}\left(\boldsymbol{u}^{1} \cdot \boldsymbol{n}^{1}+\boldsymbol{u}^{2} \cdot \boldsymbol{n}^{2}-i_{h}^{\ell}\left(\boldsymbol{u}^{1} \cdot \boldsymbol{n}^{1}+\boldsymbol{u}^{2} \cdot \boldsymbol{n}^{2}\right)\right) d \Gamma \\
\leq & \int_{\Gamma_{c}}\left(\lambda_{h}-\lambda\right)\left(\boldsymbol{u}^{1} \cdot \boldsymbol{n}^{1}+\boldsymbol{u}^{2} \cdot \boldsymbol{n}^{2}-i_{h}^{\ell}\left(\boldsymbol{u}^{1} \cdot \boldsymbol{n}^{1}+\boldsymbol{u}^{2} \cdot \boldsymbol{n}^{2}\right)\right) d \Gamma \\
& \quad+\int_{\Gamma_{c}} \lambda\left(\boldsymbol{u}^{1} \cdot \boldsymbol{n}^{1}+\boldsymbol{u}^{2} \cdot \boldsymbol{n}^{2}-i_{h}^{\ell}\left(\boldsymbol{u}^{1} \cdot \boldsymbol{n}^{1}+\boldsymbol{u}^{2} \cdot \boldsymbol{n}^{2}\right)\right) d \Gamma \\
\leq & \left\|\lambda_{h}-\lambda\right\|_{H^{-\frac{1}{2}}\left(\Gamma_{c}\right)}\left\|\boldsymbol{u}^{1} \cdot \boldsymbol{n}^{1}+\boldsymbol{u}^{2} \cdot \boldsymbol{n}^{2}-i_{h}^{\ell}\left(\boldsymbol{u}^{1} \cdot \boldsymbol{n}^{1}+\boldsymbol{u}^{2} \cdot \boldsymbol{n}^{2}\right)\right\|_{H^{\frac{1}{2}}\left(\Gamma_{c}\right)} \\
& \quad+\|\lambda\|_{L^{2}\left(\Gamma_{c}\right)}\left\|\boldsymbol{u}^{1} \cdot \boldsymbol{n}^{1}+\boldsymbol{u}^{2} \cdot \boldsymbol{n}^{2}-i_{h}^{\ell}\left(\boldsymbol{u}^{1} \cdot \boldsymbol{n}^{1}+\boldsymbol{u}^{2} \cdot \boldsymbol{n}^{2}\right)\right\|_{L^{2}\left(\Gamma_{c}\right)} \\
\leq & C(\boldsymbol{u}) h\left\|\lambda-\lambda_{h}\right\|_{H^{-\frac{1}{2}}\left(\Gamma_{c}\right)}+(C(\boldsymbol{u}))^{2} h^{\frac{3}{2}} \cdot
\end{aligned}
$$

Assembling the estimates (i)-(v) in Lemma 4.1, and using the V-ellipticity of the bilinear form $a(.,$.$) , we finally arrive at the estimate$

$$
\left\|\boldsymbol{u}-\boldsymbol{u}_{h}\right\|^{2} \leq C(\boldsymbol{u})\left(h\left\|\lambda-\lambda_{h}\right\|_{H^{-\frac{1}{2}}\left(\Gamma_{c}\right)}+h\left\|\boldsymbol{u}-\boldsymbol{u}_{h}\right\|+C(\boldsymbol{u}) h^{\frac{3}{2}}\right) .
$$

Writing $C(\boldsymbol{u}) h\left\|\boldsymbol{u}-\boldsymbol{u}_{h}\right\| \leq \gamma\left\|\boldsymbol{u}-\boldsymbol{u}_{h}\right\|^{2}+\frac{(C(\boldsymbol{u}))^{2}}{4 \gamma} h^{2}$ with $\gamma>0$ leads to the estimate announced in the lemma when $\gamma$ is chosen small enough.

Remark 4.3. 1. If $M_{h}=M_{h}^{1, \ell}$, we are not able to prove the same convergence rate as in Lemma 4.2. Nevertheless, one can easily obtain the following error bound when supposing the existence of a (small) positive $\varepsilon$ such that $\lambda \in H^{\frac{1}{2}+\varepsilon}\left(\Gamma_{c}\right)$ :

$$
\left\|\boldsymbol{u}-\boldsymbol{u}_{h}\right\|^{2} \leq C(\boldsymbol{u}) h\left\|\lambda-\lambda_{h}\right\|_{H^{-\frac{1}{2}}\left(\Gamma_{c}\right)}+C_{\varepsilon}(\lambda) h^{\frac{1}{2}+\varepsilon} .
$$

It suffices to choose $\boldsymbol{v}_{h}=I_{h} \boldsymbol{u}=\left(I_{h}^{1} \boldsymbol{u}^{1}, I_{h}^{2} \boldsymbol{u}^{2}\right)$ and $\mu_{h}=i_{h}^{\ell} \lambda$. Then terms (i),(iii) are estimated as in Lemma 4.2 and the nonpositive term (v) disappears. The terms (ii) and (iv) are added so that

$$
b\left(\lambda-\mu_{h}, \boldsymbol{u}_{h}\right) \leq C\left\|\lambda-i_{h}^{\ell} \lambda\right\|_{L^{2}\left(\Gamma_{c}\right)}\left\|\boldsymbol{u}_{h}\right\| \leq C_{\varepsilon}(\lambda) h^{\frac{1}{2}+\varepsilon}\left\|\boldsymbol{u}_{h}\right\| \leq C_{\varepsilon}^{\prime}(\lambda) h^{\frac{1}{2}+\varepsilon},
$$

where the boundedness of $\left\|\boldsymbol{u}_{h}\right\|$ resulting from the equation in 3.4 has been used.

2. The estimate obtained in (v) is suboptimal in the finite element sense. This is due to the fact that the Lagrange interpolation operator does not satisfy optimal approximation properties in the $H^{-\frac{1}{2}}\left(\Gamma_{c}\right)$-norm (see the counterexample in [13]). 
The next lemma gives an upper bound to the error committed on the multiplier.

Lemma 4.4. Let $(\boldsymbol{u}, \lambda)$ be the solution of (2.1). Suppose that $\boldsymbol{u}^{1} \in\left(H^{2}\left(\Omega^{1}\right)\right)^{2}$ and $\boldsymbol{u}^{2} \in\left(H^{2}\left(\Omega^{2}\right)\right)^{2}$. Let $\left(\boldsymbol{u}_{h}, \lambda_{h}\right)$ be the solution of 3.4 with $M_{h}=M_{h}^{1, \ell, *}$ or $M_{h}=M_{h}^{1, \ell}$ and $\ell=1$ or 2 . Then we obtain the following estimate:

$$
\left\|\lambda-\lambda_{h}\right\|_{H^{-\frac{1}{2}}\left(\Gamma_{c}\right)} \leq C\left(\left\|\boldsymbol{u}-\boldsymbol{u}_{h}\right\|+C(\boldsymbol{u}) h\right),
$$

where positive constant $C(\boldsymbol{u})$ depends linearly on $\left\|\boldsymbol{u}^{1}\right\|_{\left(H^{2}\left(\Omega^{1}\right)\right)^{2}}$ and $\left\|\boldsymbol{u}^{2}\right\|_{\left(H^{2}\left(\Omega^{2}\right)\right)^{2}}$.

Proof. Let us consider both equations incorporated in (2.1) and (3.4). Noting that $\mathbf{V}_{h} \subset \mathbf{V}$, we deduce

$$
\begin{aligned}
a\left(\boldsymbol{u}, \boldsymbol{v}_{h}\right)+b\left(\lambda, \boldsymbol{v}_{h}\right) & =L\left(\boldsymbol{v}_{h}\right), & & \forall \boldsymbol{v}_{h} \in \mathbf{V}_{h}, \\
a\left(\boldsymbol{u}_{h}, \boldsymbol{v}_{h}\right)+b\left(\lambda_{h}, \boldsymbol{v}_{h}\right) & =L\left(\boldsymbol{v}_{h}\right), & & \forall \boldsymbol{v}_{h} \in \mathbf{V}_{h} .
\end{aligned}
$$

Subtracting the equalities yields

$$
a\left(\boldsymbol{u}-\boldsymbol{u}_{h}, \boldsymbol{v}_{h}\right)+b\left(\lambda-\lambda_{h}, \boldsymbol{v}_{h}\right)=0, \quad \forall \boldsymbol{v}_{h} \in \mathbf{V}_{h}
$$

and

$$
\begin{aligned}
b\left(\lambda_{h}-\pi_{h}^{\ell} \lambda, \boldsymbol{v}_{h}\right) & =a\left(\boldsymbol{u}-\boldsymbol{u}_{h}, \boldsymbol{v}_{h}\right)+b\left(\lambda-\pi_{h}^{\ell} \lambda, \boldsymbol{v}_{h}\right) \\
& \leq C\left\|\boldsymbol{u}-\boldsymbol{u}_{h}\right\|\left\|\boldsymbol{v}_{h}\right\|+C h\|\lambda\|_{H^{\frac{1}{2}}\left(\Gamma_{c}\right)}\left\|\boldsymbol{v}_{h}\right\|, \quad \forall \boldsymbol{v}_{h} \in \mathbf{V}_{h} .
\end{aligned}
$$

Therefore the inf-sup condition (3.5) leads to

$$
\begin{aligned}
\beta\left\|\lambda_{h}-\pi_{h}^{\ell} \lambda\right\|_{H^{-\frac{1}{2}}\left(\Gamma_{c}\right)} & \leq \sup _{\boldsymbol{v}_{h} \in \mathbf{V}_{h}} \frac{b\left(\lambda_{h}-\pi_{h}^{\ell} \lambda, \boldsymbol{v}_{h}\right)}{\left\|\boldsymbol{v}_{h}\right\|} \\
& \leq C\left\|\boldsymbol{u}-\boldsymbol{u}_{h}\right\|+C h\|\lambda\|_{H^{\frac{1}{2}\left(\Gamma_{c}\right)}}
\end{aligned}
$$

A triangular inequality

$$
\left\|\lambda-\lambda_{h}\right\|_{H^{-\frac{1}{2}}\left(\Gamma_{c}\right)} \leq\left\|\lambda-\pi_{h}^{\ell} \lambda\right\|_{H^{-\frac{1}{2}}\left(\Gamma_{c}\right)}+\left\|\pi_{h}^{\ell} \lambda-\lambda_{h}\right\|_{H^{-\frac{1}{2}}\left(\Gamma_{c}\right)}
$$

together with estimates (4.4) and (4.2) ends the proof of the lemma.

We finally obtain the global result giving an upper bound of the error corresponding to our mixed finite element approximation.

Theorem 4.5. Let $(\boldsymbol{u}, \lambda)$ be the solution of (2.1). Suppose that $\boldsymbol{u}^{1} \in\left(H^{2}\left(\Omega^{1}\right)\right)^{2}$ and $\boldsymbol{u}^{2} \in\left(H^{2}\left(\Omega^{2}\right)\right)^{2}$. Let $\left(\boldsymbol{u}_{h}, \lambda_{h}\right)$ be the solution of (3.4) with $M_{h}=M_{h}^{1, \ell, *}$ and $\ell=1$ or 2 . Then we obtain the estimate

$$
\left\|\boldsymbol{u}-\boldsymbol{u}_{h}\right\|+\left\|\lambda-\lambda_{h}\right\|_{H^{-\frac{1}{2}}\left(\Gamma_{c}\right)} \leq C(\boldsymbol{u}) h^{\frac{3}{4}},
$$

where positive constant $C(\boldsymbol{u})$ depends linearly on $\left\|\boldsymbol{u}^{1}\right\|_{\left(H^{2}\left(\Omega^{1}\right)\right)^{2}}$ and $\left\|\boldsymbol{u}^{2}\right\|_{\left(H^{2}\left(\Omega^{2}\right)\right)^{2}}$.

Proof. By assembling the estimates of Lemmas 4.2 and 4.4 .

Remark 4.6. Let us enumerate more convergence results which can be stated using the regularity hypotheses of Theorem 4.5.

1. If $M_{h}=M_{h}^{1, \ell}$ and by using Lemma 4.4, we can only obtain a convergence rate close to $h^{\frac{1}{4}}$ under the additional assumption that $\varepsilon$ is close to 0 in Remark 4.3.

2. If $M_{h}=M_{h}^{0, \ell}$, then a convergence rate of $h^{\frac{3}{4}}$ can be obtained (see [17]).

3. When using a primal approach (variational inequality) and mortar finite elements (see [4]), a convergence rate of $h^{\frac{3}{4}}$ can be established (see [3] ). 
Next, we consider some other slightly stronger regularity assumptions originally introduced by Brezzi, Hager and Raviart in [5] for the Signorini problem which lead us to an optimal order of convergence.

Theorem 4.7. Let $(\boldsymbol{u}, \lambda)$ be the solution of (2.1). Suppose that $\boldsymbol{u}^{1} \in\left(H^{2}\left(\Omega^{1}\right)\right)^{2}$, $\boldsymbol{u}^{2} \in\left(H^{2}\left(\Omega^{2}\right)\right)^{2}, \boldsymbol{u}^{1} \cdot \boldsymbol{n}^{1}+\boldsymbol{u}^{2} \cdot \boldsymbol{n}^{2} \in W^{1, \infty}\left(\Gamma_{c}\right)$ and $\lambda \in L^{\infty}\left(\Gamma_{c}\right)$. Assume that the set of points of $\Gamma_{c}$ in which the change from $\boldsymbol{u}^{1} \cdot \boldsymbol{n}^{1}+\boldsymbol{u}^{2} \cdot \boldsymbol{n}^{2}<0$ to $\boldsymbol{u}^{1} \cdot \boldsymbol{n}^{1}+\boldsymbol{u}^{2} \cdot \boldsymbol{n}^{2}=0$ occurs is finite. Let $\left(\boldsymbol{u}_{h}, \lambda_{h}\right)$ be the solution of (3.4) with $M_{h}=M_{h}^{1, \ell, *}$ and $\ell=1$ or 2. Then we obtain the following estimate:

$$
\left\|\boldsymbol{u}-\boldsymbol{u}_{h}\right\|+\left\|\lambda-\lambda_{h}\right\|_{H^{-\frac{1}{2}}\left(\Gamma_{c}\right)} \leq C(\boldsymbol{u}) h
$$

where $C(\boldsymbol{u})>0$ is independent of $h$.

Proof. Let us revisit the only suboptimal convergence rate of Lemma 4.2 (i.e., part (v) coming from the term $b\left(\lambda_{h}, \boldsymbol{u}\right)$ ) and let us prove that the current hypotheses lead to the new estimate

$$
\left\|\boldsymbol{u}-\boldsymbol{u}_{h}\right\|^{2} \leq C(\boldsymbol{u})\left(h\left\|\lambda-\lambda_{h}\right\|_{H^{-\frac{1}{2}\left(\Gamma_{c}\right)}}+C(\boldsymbol{u}) h^{2}\right) .
$$

As in Lemma 4.2 , we choose $\boldsymbol{v}_{h}=I_{h} \boldsymbol{u}=\left(I_{h}^{1} \boldsymbol{u}^{1}, I_{h}^{2} \boldsymbol{u}^{2}\right)$ and $\mu_{h}=\pi_{h}^{\ell} \lambda$. The term $b\left(\lambda_{h}, \boldsymbol{u}\right)$ is estimated by using (4.3).

$$
\begin{aligned}
b\left(\lambda_{h}, \boldsymbol{u}\right) \leq & \int_{\Gamma_{c}}\left(\lambda_{h}-\lambda\right)\left(\boldsymbol{u}^{1} \cdot \boldsymbol{n}^{1}+\boldsymbol{u}^{2} \cdot \boldsymbol{n}^{2}-i_{h}^{\ell}\left(\boldsymbol{u}^{1} \cdot \boldsymbol{n}^{1}+\boldsymbol{u}^{2} \cdot \boldsymbol{n}^{2}\right)\right) d \Gamma \\
& \quad+\int_{\Gamma_{c}} \lambda\left(\boldsymbol{u}^{1} \cdot \boldsymbol{n}^{1}+\boldsymbol{u}^{2} \cdot \boldsymbol{n}^{2}-i_{h}^{\ell}\left(\boldsymbol{u}^{1} \cdot \boldsymbol{n}^{1}+\boldsymbol{u}^{2} \cdot \boldsymbol{n}^{2}\right)\right) d \Gamma \\
\leq & C(\boldsymbol{u}) h\left\|\lambda-\lambda_{h}\right\|_{H^{-\frac{1}{2}}\left(\Gamma_{c}\right)}+\int_{\Gamma_{c}} \lambda\left(\boldsymbol{u}^{1} \cdot \boldsymbol{n}^{1}+\boldsymbol{u}^{2} \cdot \boldsymbol{n}^{2}-i_{h}^{\ell}\left(\boldsymbol{u}^{1} \cdot \boldsymbol{n}^{1}+\boldsymbol{u}^{2} \cdot \boldsymbol{n}^{2}\right)\right) d \Gamma .
\end{aligned}
$$

The complementarity condition given in 1.8 -1.9 which can be also written $\lambda\left(\boldsymbol{u}^{1} \cdot \boldsymbol{n}^{1}+\boldsymbol{u}^{2} \cdot \boldsymbol{n}^{2}\right)=0$ on $\Gamma_{c}$ leads to

$$
b\left(\lambda_{h}, \boldsymbol{u}\right) \leq C(\boldsymbol{u}) h\left\|\lambda-\lambda_{h}\right\|_{H^{-\frac{1}{2}\left(\Gamma_{c}\right)}}-\int_{\Gamma_{c}} \lambda i_{h}^{\ell}\left(\boldsymbol{u}^{1} \cdot \boldsymbol{n}^{1}+\boldsymbol{u}^{2} \cdot \boldsymbol{n}^{2}\right) d \Gamma .
$$

Invoking the finite set of $N$ (1D)-meshes of the triangulation of $\Omega^{\ell}$ on $\Gamma_{c}$ in which the change from $\boldsymbol{u}^{1} \cdot \boldsymbol{n}^{1}+\boldsymbol{u}^{2} \cdot \boldsymbol{n}^{2}<0$ to $\boldsymbol{u}^{1} \cdot \boldsymbol{n}^{1}+\boldsymbol{u}^{2} \cdot \boldsymbol{n}^{2}=0$ occurs enables us to write the integral term as a finite (independent of the discretization parameter) sum of integrals on the above-mentioned (1D)-meshes denoted $T_{i}$ :

$$
\begin{aligned}
b\left(\lambda_{h}, \boldsymbol{u}\right) & \leq C(\boldsymbol{u}) h\left\|\lambda-\lambda_{h}\right\|_{H^{-\frac{1}{2}\left(\Gamma_{c}\right)}}-\sum_{i=1}^{N} \int_{T_{i}} \lambda i_{h}^{\ell}\left(\boldsymbol{u}^{1} \cdot \boldsymbol{n}^{1}+\boldsymbol{u}^{2} \cdot \boldsymbol{n}^{2}\right) d \Gamma \\
& \leq C(\boldsymbol{u}) h\left\|\lambda-\lambda_{h}\right\|_{H^{-\frac{1}{2}\left(\Gamma_{c}\right)}}+\sum_{i=1}^{N} h\|\lambda\|_{L^{\infty}\left(T_{i}\right)}\left\|i_{h}^{\ell}\left(\boldsymbol{u}^{1} \cdot \boldsymbol{n}^{1}+\boldsymbol{u}^{2} \cdot \boldsymbol{n}^{2}\right)\right\|_{L^{\infty}\left(T_{i}\right)} \\
& \leq C(\boldsymbol{u}) h\left\|\lambda-\lambda_{h}\right\|_{H^{-\frac{1}{2}\left(\Gamma_{c}\right)}}+N h^{2}\|\lambda\|_{L^{\infty}\left(\Gamma_{c}\right)}\left\|\boldsymbol{u}^{1} \cdot \boldsymbol{n}^{1}+\boldsymbol{u}^{2} \cdot \boldsymbol{n}^{2}\right\|_{W^{1, \infty}\left(\Gamma_{c}\right)} .
\end{aligned}
$$

Combining the latter result with estimates (i)-(iv) of Lemma 4.2, we obtain estimate (4.5) which together with Lemma 4.4 concludes the proof of Theorem 4.7.

Remark 4.8. This remark deals with more results which can be obtained with $M_{h}=$ $M_{h}^{1, \ell, *}$ and $\ell=1$ or 2 , but under other regularity assumptions than in Theorem 4.5 or Theorem 4.7 . 
1. If $\boldsymbol{u}^{1} \in\left(H^{2}\left(\Omega^{1}\right)\right)^{2}, \boldsymbol{u}^{2} \in\left(H^{2}\left(\Omega^{2}\right)\right)^{2}$, and if the set of points of $\Gamma_{c}$ in which the change from $\boldsymbol{u}^{1} \cdot n^{1}+\boldsymbol{u}^{2} \cdot n^{2}<0$ to $\boldsymbol{u}^{1} \cdot n^{1}+\boldsymbol{u}^{2} \cdot n^{2}=0$ occurs is finite, then

$$
\left\|\boldsymbol{u}-\boldsymbol{u}_{h}\right\|+\left\|\lambda-\lambda_{h}\right\|_{H^{-\frac{1}{2}}\left(\Gamma_{c}\right)} \leq C(\boldsymbol{u}) h|\log (h)|^{\frac{1}{2}} .
$$

This convergence can be established by using a recent estimate obtained in 2 which bounds below the integral term of (4.6) by $(C(\boldsymbol{u}))^{2} h^{2}|\log (h)|$ under the assumptions which are intermediate between those of Theorem 4.5 and those of Theorem 4.7.

2. If $\boldsymbol{u}^{1}$ and $\boldsymbol{u}^{2}$ are less regular than $H^{2}$, then the convergence rates proved for the primal approach (variational inequality) (see [2, 3]) can be extended to the present mixed analysis.

\section{Numerical StUdies}

5.1. Algebraic saddle point formulation. In this part we are interested in the matrix formulation of problem (3.4). Let $M_{h}=M_{h}^{0, \ell}$ or $M_{h}=M_{h}^{1, \ell}$ or $M_{h}=M_{h}^{1, \ell, *}$ with $\ell=1$ or 2 . We begin by noticing that $\left(\boldsymbol{u}_{h}, \lambda_{h}\right) \in \mathbf{V}_{h} \times M_{h}$ is the solution of (3.4) if and only if $\left(\boldsymbol{u}_{h}, \lambda_{h}\right)$ is a saddle-point of the Lagrangian defined on $\mathbf{V}_{h} \times M_{h}$ by

$$
\mathcal{L}\left(\boldsymbol{v}_{h}, \mu_{h}\right)=\frac{1}{2} a\left(\boldsymbol{v}_{h}, \boldsymbol{v}_{h}\right)-L\left(\boldsymbol{v}_{h}\right)+\int_{\Gamma_{c}} \mu_{h}\left(\boldsymbol{v}_{h}^{1} \cdot \boldsymbol{n}^{1}+\boldsymbol{v}_{h}^{2} \cdot \boldsymbol{n}^{2}\right) d \Gamma .
$$

In other words, we have to find $\left(\boldsymbol{u}_{h}, \lambda_{h}\right) \in \mathbf{V}_{h} \times M_{h}$ satisfying

$$
\mathcal{L}\left(\boldsymbol{u}_{h}, \mu_{h}\right) \leq \mathcal{L}\left(\boldsymbol{u}_{h}, \lambda_{h}\right) \leq \mathcal{L}\left(\boldsymbol{v}_{h}, \lambda_{h}\right), \quad \forall \boldsymbol{v}_{h} \in \mathbf{V}_{h}, \forall \mu_{h} \in M_{h} .
$$

Let $\mathbf{V}^{\ell}$ and $\mathbf{U}^{\ell}$ be the vectors associated with the nodal values of $\boldsymbol{v}_{h}^{\ell}$ and $\boldsymbol{u}_{h}^{\ell}$ respectively. Let $M$ and $\Lambda$ be the vectors corresponding to the nodal values of $\mu_{h}$ and $\lambda_{h}$ respectively when $M_{h}=M_{h}^{1, \ell}$ or $M_{h}=M_{h}^{1, \ell, *}$ with $\ell=1$ or 2 . When $M_{h}=M_{h}^{0, \ell}$ with $\ell=1$ or 2 , then $M$ and $\Lambda$ are the vectors corresponding to the values of $\mu_{h}$ and $\lambda_{h}$ respectively. With these notations, the saddle-point problem (5.1) consists of finding $\left(\begin{array}{l}\mathbf{U}^{1} \\ \mathbf{U}^{2}\end{array}\right)$ and $\Lambda$, the solution to

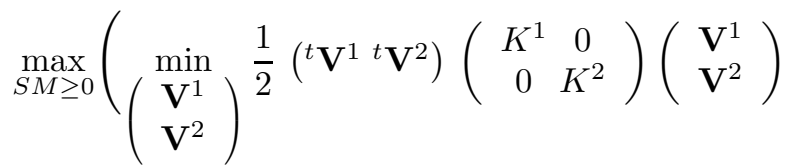

$$
\begin{aligned}
& \left.-\left({ }^{t} \mathbf{V}^{1}{ }^{t} \mathbf{V}^{2}\right)\left(\begin{array}{c}
F^{1} \\
F^{2}
\end{array}\right)+\left({ }^{t} \mathbf{V}^{1 t} \mathbf{V}^{2}\right)\left(\begin{array}{c}
C^{1} \\
0 \\
C^{2} \\
0
\end{array}\right) S M\right) .
\end{aligned}
$$

The notation $K^{\ell}$ denotes the stiffness matrix associated with $\Omega^{\ell}$ and $F^{\ell}$ is the load vector corresponding to the external loads of $\Omega^{\ell}$.

Let $m_{\ell}$ denote the number of nodes of $\Omega^{\ell}$ on $\Gamma_{c}$ and let us define $\psi_{i}^{\ell}, 1 \leq i \leq m_{\ell}$, as the basis function of $W_{h}^{1, \ell}\left(\Gamma_{c}\right)$ at node number $i$. Let $\varphi_{i}^{\ell}, 1 \leq i \leq m_{\ell}-1$, be the basis function of $W_{h}^{0, \ell}\left(\Gamma_{c}\right)$ at mesh number $i$. In (5.2), the matrix $S$ expresses the sign condition of the multipliers (3.1)-(3.3):

- if $M_{h}=M_{h}^{0, \ell}$ or $M_{h}=M_{h}^{1, \ell}$, then $S$ obviously becomes an identity matrix, 
- if $M_{h}=M_{h}^{1, \ell, *}$, then $S$ is the mass matrix associated with the mesh of $\Omega^{\ell}$ on $\Gamma_{c}$ (i.e., $S_{i, j}=\int_{\Gamma_{c}} \psi_{i}^{\ell} \psi_{j}^{\ell} d \Gamma, \quad 1 \leq i, j \leq m_{\ell}$ ).

Finally, $C^{1}$ and $C^{2}$ are the "coupling" matrices between the multipliers and the displacements. We describe hereafter these matrices in the different cases (3.1), (3.2) and (3.3):

- if $M_{h}=M_{h}^{1, \ell}$ or $M_{h}=M_{h}^{1, \ell, *}$, then $C^{\ell}$ is the mass matrix associated with the mesh of $\Omega^{\ell}$ on $\Gamma_{c}$ and $C_{i, j}^{3-\ell}=\int_{\Gamma_{c}} \psi_{i}^{3-\ell} \psi_{j}^{\ell} d \Gamma, \quad 1 \leq i \leq m_{3-\ell}, \quad 1 \leq j \leq m_{\ell} ;$

- if $M_{h}=M_{h}^{0, \ell}$, then $C_{i, j}^{\ell}=\int_{\Gamma_{c}} \psi_{i}^{\ell} \varphi_{j}^{\ell} d \Gamma, \quad 1 \leq i \leq m_{\ell}, \quad 1 \leq j \leq m_{\ell}-1$ and $C_{i, j}^{3-\ell}=\int_{\Gamma_{c}} \psi_{i}^{3-\ell} \varphi_{j}^{\ell} d \Gamma, \quad 1 \leq i \leq m_{3-\ell}, \quad 1 \leq j \leq m_{\ell}-1$.

The solution $\left(\left(\begin{array}{c}\mathbf{U}^{1} \\ \mathbf{U}^{2}\end{array}\right), \Lambda\right)$ of (5.2) satisfies the relation

$$
\left(\begin{array}{c}
\mathbf{U}^{1} \\
\mathbf{U}^{2}
\end{array}\right)=\left(\begin{array}{cc}
K^{1} & 0 \\
0 & K^{2}
\end{array}\right)^{-1}\left(\left(\begin{array}{c}
F^{1} \\
F^{2}
\end{array}\right)-\left(\begin{array}{c}
C^{1} \\
0 \\
C^{2} \\
0
\end{array}\right) S \Lambda\right) .
$$

So, setting $\Phi=S M, K=\left(\begin{array}{cc}K^{1} & 0 \\ 0 & K^{2}\end{array}\right), C=\left(\begin{array}{c}C^{1} \\ 0 \\ C^{2} \\ 0\end{array}\right), F=\left(\begin{array}{c}F^{1} \\ F^{2}\end{array}\right)$, the saddlepoint problem (5.2) can be rewritten as a minimization problem of a quadratic functional with linear inequality constraints:

$$
\min _{\Phi \geq 0}\left(\frac{1}{2}{ }^{t} \Phi^{t} C K^{-1} C \Phi-{ }^{t} \Phi^{t} C K^{-1} F+\frac{1}{2}{ }^{t} F K^{-1} F\right) .
$$

If $\bar{\Phi}$ is the solution to the minimization problem (5.4), then $\Lambda=S^{-1} \bar{\Phi}$. The displacements $\mathbf{U}^{1}$ and $\mathbf{U}^{2}$ are then obtained by (5.3). To solve the minimization problem (5.4), the iterative Frank and Wolfe algorithm (see [10, 7]) is used in the finite element code CASTEM 2000.

5.2. Examples. In this section, we study numerically the performances of the above-mentioned methods corresponding to $M_{h}=M_{h}^{0, \ell}$ or $M_{h}=M_{h}^{1, \ell}$ or $M_{h}=$ $M_{h}^{1, \ell, *}$ with $\ell=1$ or 2 . As a constitutive law, we choose a common case of the law of linear elasticity (1.21) which is Hooke's law concerning homogeneous isotropic materials:

$$
\sigma_{i j}^{\ell}=\frac{E_{\ell} \nu_{\ell}}{\left(1-2 \nu_{\ell}\right)\left(1+\nu_{\ell}\right)} \delta_{i j} \varepsilon_{k k}^{\ell}\left(\boldsymbol{u}^{\ell}\right)+\frac{E_{\ell}}{1+\nu_{\ell}} \varepsilon_{i j}^{\ell}\left(\boldsymbol{u}^{\ell}\right) \quad \text { in } \Omega^{\ell}
$$

where $E_{\ell}$ denotes Young's modulus, $\nu_{\ell}$ represents Poisson's ratio and $\delta_{i j}$ is the Kronecker symbol. The implementation is achieved using CASTEM 2000 developed at the CEA, and an HP-C160 computer has been used. 


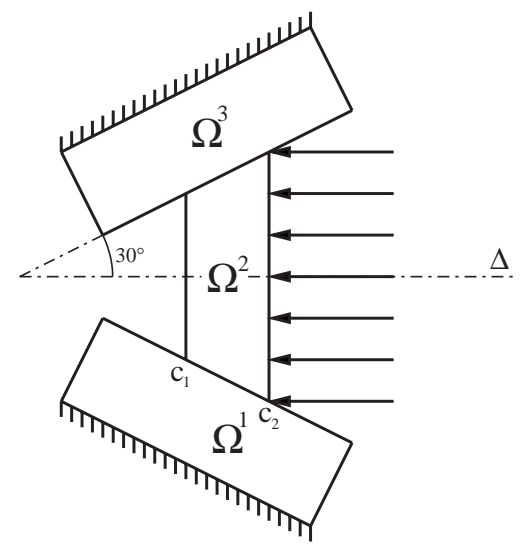

Figure 1. Set-up of the problem.

TABLE 1. The relative errors (in \%) for $\left\|\boldsymbol{u}-\boldsymbol{u}_{h}\right\|$

\begin{tabular}{|c||c|c|c|c|c|}
\hline $\begin{array}{c}\text { Number of elements } \\
\text { in contact }\end{array}$ & 1 & 2 & 4 & 8 & 16 \\
\hline \hline$M_{h}=M_{h}^{1,1, *}=M_{h}^{1,2, *}$ & 41,630 & 28,562 & 18,926 & 12,104 & 7,403 \\
\hline$M_{h}=M_{h}^{1,1}=M_{h}^{1,2}$ & 41,872 & 28,562 & 18,926 & 12,101 & 7,381 \\
\hline$M_{h}=M_{h}^{0,1}=M_{h}^{0,2}$ & 49,027 & 29,072 & 18,884 & 11,882 & 7,337 \\
\hline
\end{tabular}

TABLE 2. The relative errors (in \%) for $\left\|\boldsymbol{u}-\boldsymbol{u}_{h}\right\|_{\left(L^{2}\left(\Omega^{1} \cup \Omega^{2}\right)\right)^{2}}$

\begin{tabular}{|c||c|c|c|c|c|}
\hline $\begin{array}{c}\text { Number of elements } \\
\text { in contact }\end{array}$ & 1 & 2 & 4 & 8 & 16 \\
\hline \hline$M_{h}=M_{h}^{1,1, *}=M_{h}^{1,2, *}$ & 21,973 & 12,014 & 5,996 & 2,700 & 1,089 \\
\hline$M_{h}=M_{h}^{1,1}=M_{h}^{1,2}$ & 21,794 & 12,014 & 5,996 & 2,700 & 1,088 \\
\hline$M_{h}=M_{h}^{0,1}=M_{h}^{0,2}$ & 12,050 & 9,694 & 5,249 & 2,337 & 0,930 \\
\hline
\end{tabular}

TABLE 3. The relative errors (in \%) for $\left\|\lambda-\lambda_{h}\right\|_{L^{2}\left(\Gamma_{c}\right)}$

\begin{tabular}{|c||c|c|c|c|c|}
\hline $\begin{array}{c}\text { Number of elements } \\
\text { in contact }\end{array}$ & 1 & 2 & 4 & 8 & 16 \\
\hline \hline$M_{h}=M_{h}^{1,1, *}=M_{h}^{1,2, *}$ & 40,369 & 38,829 & 35,773 & 32,330 & 27,827 \\
\hline$M_{h}=M_{h}^{1,1}=M_{h}^{1,2}$ & 40,485 & 38,829 & 35,773 & 32,317 & 27,692 \\
\hline$M_{h}=M_{h}^{0,1}=M_{h}^{0,2}$ & 42,303 & 32,784 & 28,381 & 23,471 & 18,097 \\
\hline
\end{tabular}

5.2.1. Example of a tapered joint. First we consider the two-dimensional plane strain problem involving the structure depicted in Figure 1. The rectangular bodies $\Omega^{1}$ and $\Omega^{3}$ are identical: their dimensions are $1 \mathrm{~mm} \times 3 \mathrm{~mm}, E_{1}=E_{3}=15 G p a$ is Young's modulus and $\nu_{1}=\nu_{3}=0.4$ are Poisson's ratios. Both bodies are clamped on their dashed parts. Young's modulus and Poisson's ratio for $\Omega^{2}$ are $30 G p a$ and 0.3 respectively. The length of the segment $\left[\boldsymbol{c}_{1}, \boldsymbol{c}_{2}\right]$ (centered on the side of $\Omega^{1}$ ) is 
TABLE 4. The relative errors (in \%) for $\left\|\boldsymbol{u}-\boldsymbol{u}_{h}\right\|$

\begin{tabular}{|c|c|c|c|}
\hline $\begin{array}{l}\text { Number of elements } \\
\text { of } \Omega^{1} \text { and } \Omega^{2} \text { in contact }\end{array}$ & $\begin{array}{l}\Omega^{1}: 2 \\
\Omega^{2}: 3\end{array}$ & $\begin{array}{l}\Omega^{1}: 4 \\
\Omega^{2}: 6\end{array}$ & $\begin{array}{l}\Omega^{1}: 8 \\
\Omega^{2}: 12\end{array}$ \\
\hline$\overline{M_{h}=M_{h}^{1,1, *}}$ & 21,086 & 13,560 & 8,278 \\
\hline$M_{h}=M_{h}^{1,1}$ & 20,951 & 13,661 & 8,475 \\
\hline$M_{h}=M_{h}^{0,1}$ & 23,872 & 17,742 & 13,398 \\
\hline
\end{tabular}

TABLE 5. The relative errors (in \%) for $\left\|\boldsymbol{u}-\boldsymbol{u}_{h}\right\|_{\left(L^{2}\left(\Omega^{1} \cup \Omega^{2}\right)\right)^{2}}$

\begin{tabular}{|c|c|c|c|}
\hline $\begin{array}{l}\text { Number of elements } \\
\text { of } \Omega^{1} \text { and } \Omega^{2} \text { in contact }\end{array}$ & $\begin{array}{l}\Omega^{1}: 2 \\
\Omega^{2}: 3\end{array}$ & $\begin{array}{l}\Omega^{1}: 4 \\
\Omega^{2}: 6\end{array}$ & $\begin{array}{l}\Omega^{1}: 8 \\
\Omega^{2}: 12\end{array}$ \\
\hline$M_{h}=M_{h}^{1,1, *}$ & 7,441 & 3,459 & 1,409 \\
\hline$M_{h}=M_{h}^{1,1}$ & 6,794 & 3,036 & 1,217 \\
\hline$M_{h}=M_{h}^{0,1}$ & 2,820 & 1,165 & 0,493 \\
\hline
\end{tabular}

TABLE 6. The relative errors (in \%) for $\left\|\lambda-\lambda_{h}\right\|_{L^{2}\left(\Gamma_{c}\right)}$

\begin{tabular}{|c||c|c|c|}
\hline $\begin{array}{c}\text { Number of elements } \\
\text { of } \Omega^{1} \text { and } \Omega^{2} \text { in contact }\end{array}$ & $\Omega^{1}: 2$ & $\Omega^{1}: 4$ & $\Omega^{1}: 8$ \\
$\Omega^{2}: 6$ & $\Omega^{2}: 12$ \\
\hline \hline$M_{h}=M_{h}^{1,1, *}$ & 35,387 & 31,896 & 27,542 \\
\hline$M_{h}=M_{h}^{1,1} 4$ & 34,205 & 30,479 & 26,039 \\
\hline$M_{h}=M_{h}^{0,1}$ & 25,952 & 21,826 & 16,748 \\
\hline
\end{tabular}

$1 \mathrm{~mm}$ and $\Omega^{2}$ is submitted to loads of $5 d a \mathrm{~N} / \mathrm{mm}^{2}$ on its side of length $3 \mathrm{~mm}$ (see Figure 1). No body forces are applied. Finally, the computations are performed on one half of the structure as shown by the axis of symmetry $\Delta$.

To judge the performances of the various methods, we compute the relative errors of $\left\|\boldsymbol{u}-\boldsymbol{u}_{h}\right\|,\left\|\boldsymbol{u}-\boldsymbol{u}_{h}\right\|_{\left(L^{2}\left(\Omega^{1} \cup \Omega^{2}\right)\right)^{2}}$ and $\left\|\lambda-\lambda_{h}\right\|_{L^{2}\left(\Gamma_{c}\right)}$. Since the exact solution $(\boldsymbol{u}, \lambda)$ is not available, we use a reference solution obtained with a very fine mesh (as fine as the computation allows).

We begin with the study in which the meshes match on the contact zone (i.e., the nodes coming from the discretizations of $\Omega^{1}$ and $\Omega^{2}$ coincide on $\left.\left[\boldsymbol{c}_{1}, \boldsymbol{c}_{2}\right]\right)$. In this case the reference mesh comprises 45056 triangular elements, 23010 nodes and 64 elements on the contact zone. Then we compute the various errors as a function of the number of elements in contact (by using a family of nested meshes) for the different methods. The results are reported in Tables 1-3 and Figures 2-4. We then consider the case of nonmatching meshes on the contact zone. In such a case the reference mesh comprises 21248 triangular elements, 10938 nodes, 32 meshes of $\Omega^{1}$ and 48 of $\Omega^{2}$ on the contact zone. The errors are given in Tables 4-6.

We notice that the same error and convergence rates of $\left\|\boldsymbol{u}-\boldsymbol{u}_{h}\right\|$ occur for the three methods (a rate of around 0.7) in the matching case in Table 1. In the case of nonmatching meshes, the third method is less satisfactory than the other two (see Table 4 ). The convergence rates of $\left\|\boldsymbol{u}-\boldsymbol{u}_{h}\right\|_{\left(L^{2}\left(\Omega^{1} \cup \Omega^{2}\right)\right)^{2}}$ (see also the appendix for some theoretical studies) in Tables 2,5 are similar for the three 


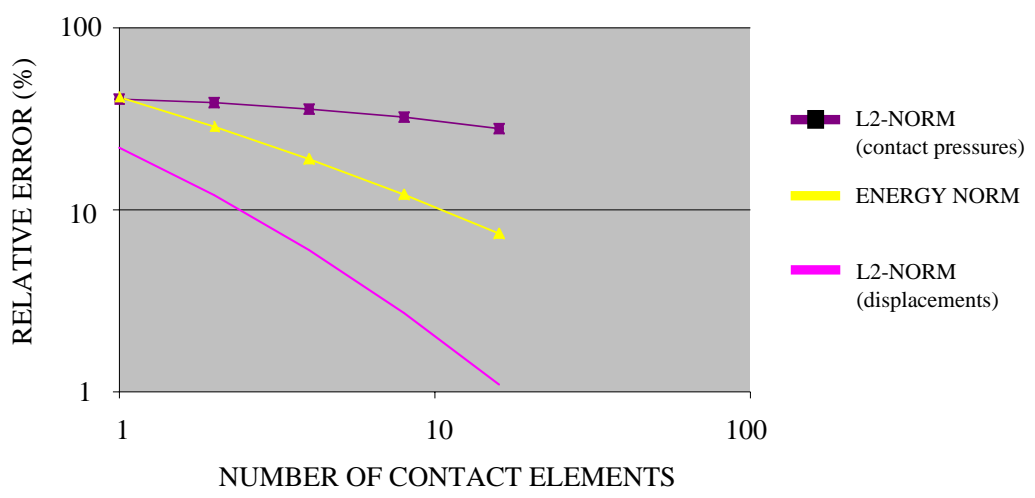

Figure 2. The relative errors (in \%) when $M_{h}=M_{h}^{1,1, *}=M_{h}^{1,2, *}$

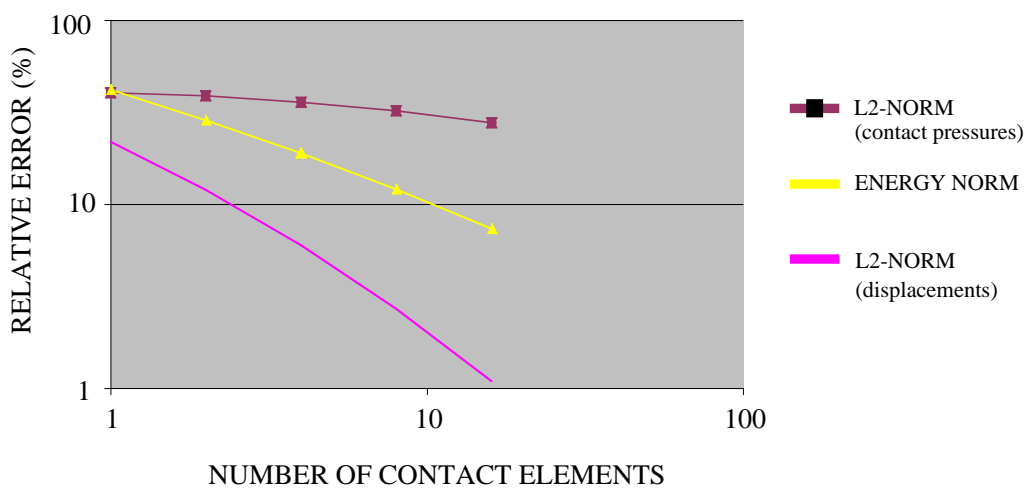

Figure 3. The relative errors (in \%) when $M_{h}=M_{h}^{1,1}=M_{h}^{1,2}$

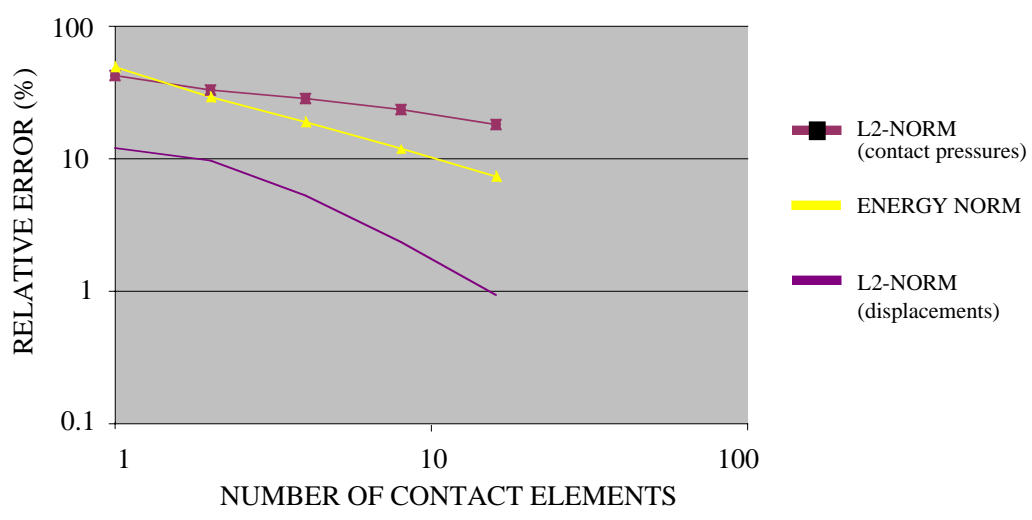

Figure 4. The relative errors (in \%) when $M_{h}=M_{h}^{0,1}=M_{h}^{0,2}$

methods (a convergence rate of around 1.3). Note that the error remains lower for the third approach than for the other two in the nonmatching case (Table 5 ). Finally, the convergence rates of $\left\|\lambda-\lambda_{h}\right\|_{L^{2}\left(\Gamma_{c}\right)}$ (see also the appendix for some further explanation) are reported in Tables 3,6 and the piecewise constant 


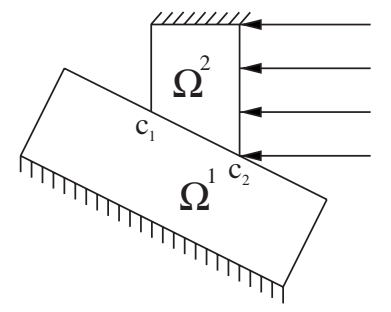

Figure 5. Set-up of the problem.

TABLE 7. The relative errors (in \%) for $\left\|\boldsymbol{u}-\boldsymbol{u}_{h}\right\|$

\begin{tabular}{|c||c|c|c|c|c|}
\hline $\begin{array}{c}\text { Number of elements } \\
\text { in contact }\end{array}$ & 1 & 2 & 4 & 8 & 16 \\
\hline \hline$M_{h}=M_{h}^{1,1, *}=M_{h}^{1,2, *}$ & 46,079 & 30,237 & 18,496 & 10,679 & 5,882 \\
\hline$M_{h}=M_{h}^{1,1}=M_{h}^{1,2}$ & 46,079 & 30,237 & 18,496 & 10,679 & 5,882 \\
\hline$M_{h}=M_{h}^{0,1}=M_{h}^{0,2}$ & 46,627 & 30,312 & 18,513 & 10,678 & 5,882 \\
\hline
\end{tabular}

TABLE 8. The relative errors (in \%) for $\left\|\boldsymbol{u}-\boldsymbol{u}_{h}\right\|_{\left(L^{2}\left(\Omega^{1} \cup \Omega^{2}\right)\right)^{2}}$

\begin{tabular}{|c||c|c|c|c|c|}
\hline $\begin{array}{c}\text { Number of elements } \\
\text { in contact }\end{array}$ & 1 & 2 & 4 & 8 & 16 \\
\hline \hline$M_{h}=M_{h}^{1,1, *}=M_{h}^{1,2, *}$ & 28,912 & 14,900 & 6,349 & 2,272 & 0,712 \\
\hline$M_{h}=M_{h}^{1,1}=M_{h}^{1,2}$ & 28,912 & 14,900 & 6,349 & 2,272 & 0,712 \\
\hline$M_{h}=M_{h}^{0,1}=M_{h}^{0,2}$ & 26,837 & 14,756 & 6,340 & 2,271 & 0,711 \\
\hline
\end{tabular}

TABLE 9. The relative errors (in \%) for $\left\|\lambda-\lambda_{h}\right\|_{L^{2}\left(\Gamma_{c}\right)}$

\begin{tabular}{|c||c|c|c|c|c|}
\hline $\begin{array}{c}\text { Number of elements } \\
\text { in contact }\end{array}$ & 1 & 2 & 4 & 8 & 16 \\
\hline \hline$M_{h}=M_{h}^{1,1, *}=M_{h}^{1,2, *}$ & 10,020 & 11,805 & 9,627 & 7,139 & 5,423 \\
\hline$M_{h}=M_{h}^{1,1}=M_{h}^{1,2}$ & 10,020 & 11,805 & 9,627 & 7,139 & 5,423 \\
\hline$M_{h}=M_{h}^{0,1}=M_{h}^{0,2}$ & 18,744 & 9,336 & 9,003 & 6,549 & 4,783 \\
\hline
\end{tabular}

multipliers approach seems to give the best results: the rate is around 0.35 instead of 0.2 for the two other methods.

Next, we replace the symmetry conditions of the problem depicted in Figure 1 by embedding conditions as suggested in Figure 5. Consequently the bilinear form $a(.,$.$) is \mathbf{V}$-elliptic which corresponds with the assumptions of the theoretical part whereas it was only $\mathbf{K}$-elliptic in the former case (see Remark 2.2 for the definition of $\mathbf{K}$ and [11], Theorem 6.3 for conditions leading to $\mathbf{K}$-ellipticity). The same convergence studies (using the same meshes) as in the previous case are performed and the corresponding results are reported in Tables 7-12.

Let us remark that both approaches handling with continuous piecewise linear multipliers lead to the same results in the case of matching meshes in Tables 7-9 
TABLE 10. The relative errors (in \%) for $\left\|\boldsymbol{u}-\boldsymbol{u}_{h}\right\|$

\begin{tabular}{|c||c|c|c|}
\hline $\begin{array}{c}\text { Number of elements } \\
\text { of } \Omega^{1} \text { and } \Omega^{2} \text { in contact }\end{array}$ & $\Omega^{1}: 2$ & $\Omega^{1}: 4$ & $\Omega^{1}: 8$ \\
\hline \hline$M_{h}=M_{h}^{1,1, *}$ & 24,912 & 15,025 & 8,311 \\
\hline$M_{h}=M_{h}^{1,1}$ & 24,717 & 14,768 & 8,250 \\
\hline$M_{h}=M_{h}^{0,1}$ & 25,941 & 17,116 & 11,611 \\
\hline
\end{tabular}

TABLE 11. The relative errors (in \%) for $\left\|\boldsymbol{u}-\boldsymbol{u}_{h}\right\|_{\left(L^{2}\left(\Omega^{1} \cup \Omega^{2}\right)\right)^{2}}$

\begin{tabular}{|c||c|c|c|}
\hline $\begin{array}{c}\text { Number of elements } \\
\text { of } \Omega^{1} \text { and } \Omega^{2} \text { in contact }\end{array}$ & $\Omega^{1}: 2$ & $\Omega^{1}: 4$ & $\Omega^{1}: 8$ \\
\hline \hline$M_{h}=M_{h}^{1,1, *}$ & 11,834 & 4,681 & 1,531 \\
\hline$M_{h}=M_{h}^{1,1}$ & 11,737 & 4,587 & 1,521 \\
\hline$M_{h}=M_{h}^{0,1}$ & 11,104 & 4,492 & 1,511 \\
\hline
\end{tabular}

TABLE 12. The relative errors (in \%) for $\left\|\lambda-\lambda_{h}\right\|_{L^{2}\left(\Gamma_{c}\right)}$

\begin{tabular}{|c||c|c|c|}
\hline $\begin{array}{c}\text { Number of elements } \\
\text { of } \Omega^{1} \text { and } \Omega^{2} \text { in contact }\end{array}$ & $\Omega^{1}: 2$ & $\Omega^{1}: 4$ & $\Omega^{1}: 8$ \\
\hline \hline$M_{h}=M_{h}^{1,1, *}$ & 12,952 & 10,053 & 8,135 \\
\hline$M_{h}=M_{h}^{1,1}$ & 12,178 & 9,132 & 7,297 \\
\hline$M_{h}=M_{h}^{0,1}$ & 5,592 & 6,068 & 4,975 \\
\hline
\end{tabular}

and a difference of only $10^{-8 \%}$ can be perceived. The convergence rates are globally a little bit greater than the previous ones. Note that the error corresponding to the multipliers in Tables 9 and 12 has been divided by three or four in comparison with the previous computations. This surprising result can be explained by noticing that both problems are quite different from a mechanical point of view.

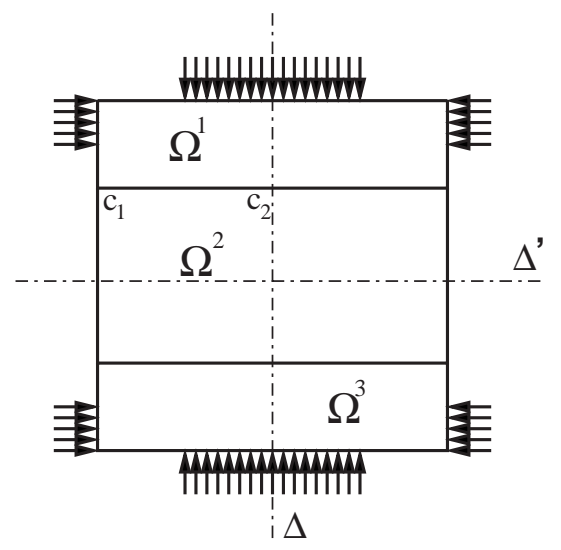

Figure 6. Set-up of the problem. 
5.2.2. Second example. As a second example, we choose the configuration of Figure 6 involving three bodies $\Omega^{1}, \Omega^{2}$ and $\Omega^{3}$ where $\Omega^{1}$ and $\Omega^{3}$ have the same geometrical and material characteristics. The dimensions of $\Omega^{1}$ and $\Omega^{2}$ are $2 \mathrm{~mm} \times 0.5 \mathrm{~mm}$ and $2 \mathrm{~mm} \times 1 \mathrm{~mm}$; Young's moduli are 80Gpa and 200Gpa and Poisson's ratios are 0.3 and 0.3 , respectively. The bodies are submitted to tractions of $10 \mathrm{daN} / \mathrm{mm}^{2}$ (see Figure 6) and no body forces are assumed. Due to both symmetry axes $\Delta$ and $\Delta^{\prime}$, we consider only a quarter of the problem. To avoid singularities arising from adjacent Dirichlet and Neumann conditions, we have removed the embedding conditions. Moreover the loads have been oriented in order to obtain a separation of the bodies. Such a configuration corresponds to a K-elliptic case (see 11], Theorem 6.3 ); it nevertheless allows the comparison between the three methods.

As in the first example, we compute the different errors as a function of the number of elements in contact for the different methods. The case of matching meshes involves a reference mesh comprising 24576 triangular elements, 12610 nodes and 64 elements on the contact zone: the results are shown in Tables 13-15 and Figures 7-9. In the nonmatching case, the reference mesh comprises 11264 triangular elements, 5874 nodes, 64 meshes of $\Omega^{1}$ and 96 of $\Omega^{2}$ on the contact zone. The approximation errors are shown in Tables 16-18.

Following this test, we observe that the three methods yield globally similar results. The only small significant difference is that the multiplier converges faster for the first approach when nonmatching meshes are used (in Table 18). It can be noted that this example is more regular than the first one because separation occurs on the contact zone $\left[\boldsymbol{c}_{1}, \boldsymbol{c}_{2}\right]$ and the multiplier remains bounded. The convergence

TABLE 13. The relative errors (in \%) for $\left\|\boldsymbol{u}-\boldsymbol{u}_{h}\right\|$

\begin{tabular}{|c||c|c|c|c|}
\hline $\begin{array}{c}\text { Number of elements } \\
\text { in contact }\end{array}$ & 2 & 4 & 8 & 16 \\
\hline \hline$M_{h}=M_{h}^{1,1, *}=M_{h}^{1,2, *}$ & 27,321 & 16,936 & 10,157 & 5,640 \\
\hline$M_{h}=M_{h}^{1,1}=M_{h}^{1,2}$ & 27,556 & 16,946 & 10,161 & 5,625 \\
\hline$M_{h}=M_{h}^{0,1}=M_{h}^{0,2}$ & 27,269 & 16,574 & 10,159 & 5,640 \\
\hline
\end{tabular}

TABLE 14. The relative errors (in \%) for $\left\|\boldsymbol{u}-\boldsymbol{u}_{h}\right\|_{\left(L^{2}\left(\Omega^{1} \cup \Omega^{2}\right)\right)^{2}}$

\begin{tabular}{|c||c|c|c|c|}
\hline $\begin{array}{c}\text { Number of elements } \\
\text { in contact }\end{array}$ & 2 & 4 & 8 & 16 \\
\hline \hline$M_{h}=M_{h}^{1,1, *}=M_{h}^{1,2, *}$ & 46,908 & 31,092 & 15,858 & 5,829 \\
\hline$M_{h}=M_{h}^{1,1}=M_{h}^{1,2}$ & 42,410 & 27,252 & 15,766 & 5,730 \\
\hline$M_{h}=M_{h}^{0,1}=M_{h}^{0,2}$ & 46,862 & 29,770 & 15,603 & 5,855 \\
\hline
\end{tabular}

TABLE 15. The relative errors (in \%) for $\left\|\lambda-\lambda_{h}\right\|_{L^{2}\left(\Gamma_{c}\right)}$

\begin{tabular}{|c||c|c|c|c|}
\hline Number of elements in contact & 2 & 4 & 8 & 16 \\
\hline \hline$M_{h}=M_{h}^{1,1, *}=M_{h}^{1,2, *}$ & 11,980 & 9,716 & 3,510 & 2,047 \\
\hline$M_{h}=M_{h}^{1,1}=M_{h}^{1,2}$ & 13,275 & 8,355 & 3,552 & 1,977 \\
\hline$M_{h}=M_{h}^{0,1}=M_{h}^{0,2}$ & 11,658 & 6,102 & 3,400 & 2,068 \\
\hline
\end{tabular}




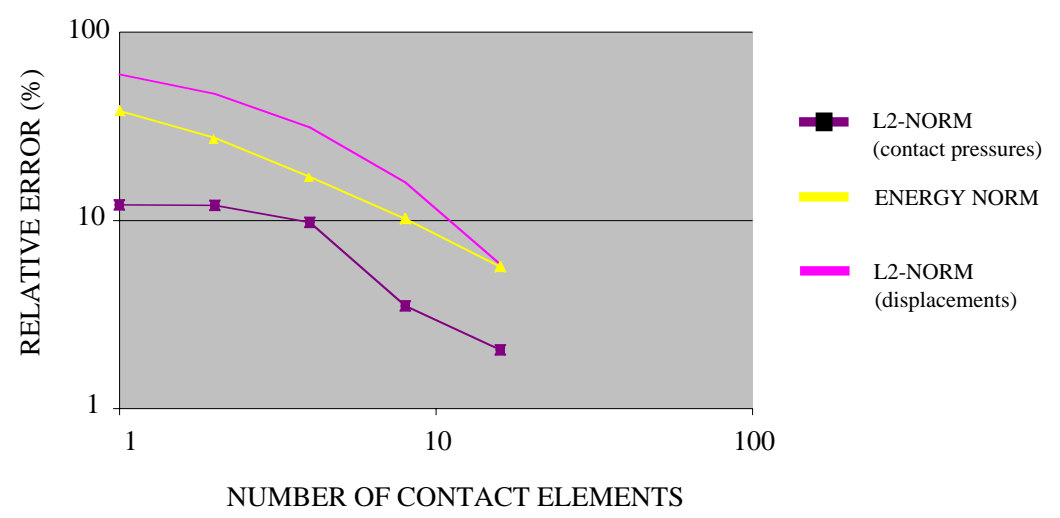

Figure 7. The relative errors (in \%) when $M_{h}=M_{h}^{1,1, *}=M_{h}^{1,2, *}$

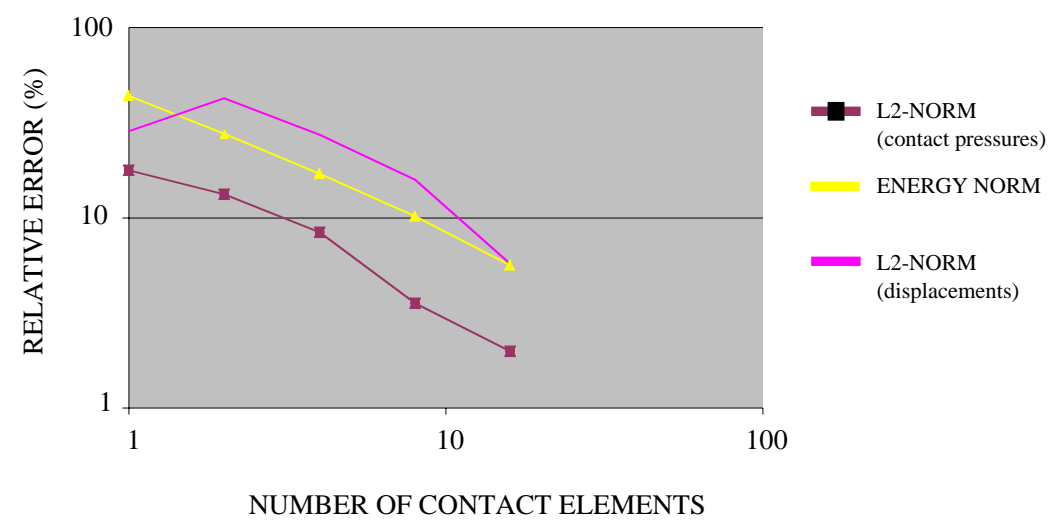

FiguRE 8. The relative errors (in \%) when $M_{h}=M_{h}^{1,1}=M_{h}^{1,2}$

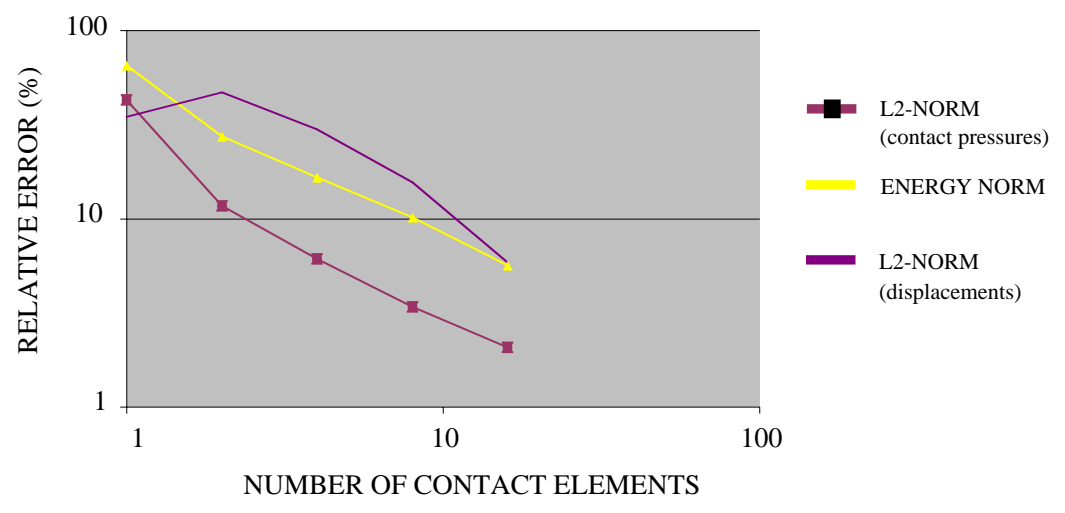

Figure 9. The relative errors (in \%) when $M_{h}=M_{h}^{0,1}=M_{h}^{0,2}$

rates obtained (around $0.85-0.9$ for the $H 1$-norm, 1.45 for the $L 2$-norm on the displacements and always more than 0.5 for the multipliers) are then greater than for the tapered joint. 
TABLE 16. The relative errors (in \%) for $\left\|\boldsymbol{u}-\boldsymbol{u}_{h}\right\|$

\begin{tabular}{|c|c|c|c|c|}
\hline $\begin{array}{l}\text { Number of elements } \\
\text { of } \Omega^{1} \text { and } \Omega^{2} \text { in contact }\end{array}$ & $\begin{array}{l}\Omega^{1}: 2 \\
\Omega^{2}: 3\end{array}$ & $\begin{array}{l}\Omega^{1}: 4 \\
\Omega^{2}: 6\end{array}$ & $\begin{array}{l}\Omega^{1}: 8 \\
\Omega^{2}: 12\end{array}$ & $\begin{array}{l}\Omega^{1}: 16 \\
\Omega^{2}: 24\end{array}$ \\
\hline$\overline{M_{h}=M_{h}^{1,1, *}}$ & 23,775 & 14,845 & 8,612 & 4,583 \\
\hline$M_{h}=M_{h}^{1,1}$ & 24,485 & 15,077 & 8,624 & 4,590 \\
\hline$M_{h}=M_{h}^{0,1}$ & 23,127 & 15,382 & 9,191 & 5,096 \\
\hline
\end{tabular}

TABLE 17. The relative errors (in \%) for $\left\|\boldsymbol{u}-\boldsymbol{u}_{h}\right\|_{\left(L^{2}\left(\Omega^{1} \cup \Omega^{2}\right)\right)^{2}}$

\begin{tabular}{|c|c|c|c|c|}
\hline $\begin{array}{l}\text { Number of elements } \\
\text { of } \Omega^{1} \text { and } \Omega^{2} \text { in contact }\end{array}$ & $\begin{array}{l}\Omega^{1}: 2 \\
\Omega^{2}: 3\end{array}$ & $\begin{array}{l}\Omega^{1}: 4 \\
\Omega^{2}: 6\end{array}$ & $\begin{array}{l}\Omega^{1}: 8 \\
\Omega^{2}: 12\end{array}$ & $\begin{array}{l}\Omega^{1}: 16 \\
\Omega^{2}: 24\end{array}$ \\
\hline$M_{h}=M_{h}^{1,1, *}$ & 36,756 & 22,852 & 8,733 & 2,727 \\
\hline$M_{h}=M_{h}^{1,1}$ & 31,488 & 18,582 & 8,308 & 2,594 \\
\hline$M_{h}=M_{h}^{0,1}$ & 37,481 & 27,156 & 10,890 & 3,654 \\
\hline
\end{tabular}

TABLE 18. The relative errors (in \%) for $\left\|\lambda-\lambda_{h}\right\|_{L^{2}\left(\Gamma_{c}\right)}$

\begin{tabular}{|c||c|c|c|c|}
\hline $\begin{array}{c}\text { Number of elements } \\
\text { of } \Omega^{1} \text { and } \Omega^{2} \text { in contact }\end{array}$ & $\Omega^{1}: 2$ & $\Omega^{1}: 4$ & $\Omega^{1}: 8$ & $\Omega^{1}: 16$ \\
\hline \hline$M_{h}=M_{h}^{1,1, *}$ & 13,403 & 7,001 & 2,025 & 1,335 \\
\hline$M_{h}=M_{h}^{1,1}$ & 13,114 & 6,544 & 2,164 & 1,828 \\
\hline$M_{h}=M_{h}^{0,1}$ & 13,279 & 7,188 & 3,127 & 2,216 \\
\hline
\end{tabular}

From the numerical examples, we have come to the conclusion that the differences between the various mixed finite element approaches are not very significant and that the three methods studied lead to satisfactory results.

\section{Appendix}

In this appendix, we prove some error estimates in the case $M_{h}=M_{h}^{1, \ell, *}$ corresponding to the convergence rates obtained numerically in section 5.2: $\left\|\lambda-\lambda_{h}\right\|_{L^{2}\left(\Gamma_{c}\right)}$ and $\left\|\boldsymbol{u}-\boldsymbol{u}_{h}\right\|_{\left(L^{2}\left(\Omega^{1} \cup \Omega^{2}\right)\right)^{2}}$. We begin with the following proposition in which we suppose that both families of discretizations $\mathcal{T}_{h}^{\ell}$ on $\Omega^{\ell}$ are uniformly regular and that $h_{1}$ and $h_{2}$ are of the same order (this is the case for both numerical examples in section 5.2).

Proposition 6.1. Let $(\boldsymbol{u}, \lambda)$ be the solution of (2.1). Let $\left(\boldsymbol{u}_{h}, \lambda_{h}\right)$ be the solution of (3.4) with $M_{h}=M_{h}^{1, \ell, *}$ and $\ell=1$ or 2 . Assume that the regularity assumptions of Theorem 4.5 hold and that both families of discretizations $\mathcal{T}_{h}^{\ell}$ on $\Omega^{\ell}$ are uniformly regular. Suppose that $h_{1} / h_{2}$ and $h_{2} / h_{1}$ are bounded. Then there is a constant $C(\boldsymbol{u})$ independent of $h$ such that

$$
\left\|\lambda-\lambda_{h}\right\|_{L^{2}\left(\Gamma_{c}\right)} \leq C(\boldsymbol{u}) h^{\frac{1}{4}} .
$$


In addition, suppose that the regularity assumptions of Theorem 4.7 hold. Then there is a constant $C(\boldsymbol{u})$ independent of $h$ such that

$$
\left\|\lambda-\lambda_{h}\right\|_{L^{2}\left(\Gamma_{c}\right)} \leq C(\boldsymbol{u}) h^{\frac{1}{2}} .
$$

Proof. A triangular inequality together with an inverse inequality and the approximation property of the projection operator $\pi_{h}^{\ell}$ yield

$$
\begin{aligned}
\left\|\lambda-\lambda_{h}\right\|_{L^{2}\left(\Gamma_{c}\right)} & \leq\left\|\lambda-\pi_{h}^{\ell} \lambda\right\|_{L^{2}\left(\Gamma_{c}\right)}+\left\|\pi_{h}^{\ell} \lambda-\lambda_{h}\right\|_{L^{2}\left(\Gamma_{c}\right)} \\
& \leq C(\boldsymbol{u}) h^{\frac{1}{2}}+h_{\ell}^{-\frac{1}{2}}\left\|\pi_{h}^{\ell} \lambda-\lambda_{h}\right\|_{H^{-\frac{1}{2}}\left(\Gamma_{c}\right)} \\
& \leq C(\boldsymbol{u}) h^{\frac{1}{2}}+h_{\ell}^{-\frac{1}{2}}\left(\left\|\pi_{h}^{\ell} \lambda-\lambda\right\|_{H^{-\frac{1}{2}}\left(\Gamma_{c}\right)}+\left\|\lambda-\lambda_{h}\right\|_{H^{-\frac{1}{2}}\left(\Gamma_{c}\right)}\right) \\
& \leq C(\boldsymbol{u}) h^{\frac{1}{2}}+h^{-\frac{1}{2}}\left\|\lambda-\lambda_{h}\right\|_{H^{-\frac{1}{2}}\left(\Gamma_{c}\right)},
\end{aligned}
$$

which ends the proof of the proposition.

By using techniques issued from [18, we establish a convergence rate in the $L^{2}$ norm for the contact problem applied to the displacements when $M_{h}=M_{h}^{1,1, *}=$ $M_{h}^{1,2, *}$ (matching meshes). We begin with a property proved in [12], Lemma 4.1 which can be stated as follows: let $\left(\boldsymbol{u}_{h}, \lambda_{h}\right)$ be the solution of problem (3.4) with $M_{h}=M_{h}^{1,1, *}=M_{h}^{1,2, *}$. Then $\boldsymbol{u}_{h}$ is the solution of the variational inequality

$$
\boldsymbol{u}_{h} \in \mathbf{K}_{h}, \quad a\left(\boldsymbol{u}_{h}, \boldsymbol{v}_{h}-\boldsymbol{u}_{h}\right) \geq L\left(\boldsymbol{v}_{h}-\boldsymbol{u}_{h}\right), \quad \forall \boldsymbol{v}_{h} \in \mathbf{K}_{h}
$$

where

$$
\mathbf{K}_{h}=\left\{\boldsymbol{v}_{h}=\left(\boldsymbol{v}_{h}^{1}, \boldsymbol{v}_{h}^{2}\right) \in \mathbf{V}_{h}, \quad \boldsymbol{v}_{h}^{1} \cdot \boldsymbol{n}^{1}+\boldsymbol{v}_{h}^{2} \cdot \boldsymbol{n}^{2} \leq 0 \text { on } \Gamma_{c}\right\} .
$$

It is straightforward that $\mathbf{K}_{h} \subset \mathbf{K}$ (see Remark 2.2 for the definition of $\mathbf{K}$ ). The notation $T\left(\mathbf{K}, \boldsymbol{u}_{h}\right)$ stands for the set of the $\boldsymbol{v} \in \mathbf{V}$ for which a $\tau_{0}>0$ and a function $f:\left[0, \tau_{0}\right] \rightarrow \mathbf{V}$ exist satisfying

$$
\|f(\tau)\|=o(\tau) \quad \text { and } \quad \boldsymbol{u}_{h}+\tau \boldsymbol{v}+f(\tau) \in \mathbf{K} \quad \text { for } \quad 0<\tau<\tau_{0} .
$$

Note that $\tau_{0}$ and $f$ depend on $\boldsymbol{v}$ in the previous definition. Set

$$
\mathcal{K}=\left\{\boldsymbol{v} \in \overline{T\left(\mathbf{K}, \boldsymbol{u}_{h}\right)}, \quad b\left(\lambda, \boldsymbol{u}_{h}+\boldsymbol{v}\right) \geq 0\right\},
$$

where $\overline{T\left(\mathbf{K}, \boldsymbol{u}_{h}\right)}$ denotes the closure of $T\left(\mathbf{K}, \boldsymbol{u}_{h}\right)$. It can be proved (see [18]) that $\mathcal{K}$ is a nonempty closed convex cone and that

$$
\mathcal{K}+\boldsymbol{u}-\boldsymbol{u}_{h} \subset \mathcal{K} .
$$

Given $\boldsymbol{f} \in\left(L^{2}\left(\Omega^{1} \cup \Omega^{2}\right)\right)^{2}$, we consider the problem of finding $\boldsymbol{z} \in \mathcal{K}$ so that

$$
\boldsymbol{z} \in \mathcal{K}, \quad a(\boldsymbol{z}, \boldsymbol{v}-\boldsymbol{z}) \geq(\boldsymbol{f}, \boldsymbol{v}-\boldsymbol{z})_{\left(L^{2}\left(\Omega^{1} \cup \Omega^{2}\right)\right)^{2}}, \quad \forall \boldsymbol{v} \in \mathcal{K},
$$

which admits a unique solution according to Stampacchia's Theorem.

Problem (6.3) is said to be regular if, for every $f \in\left(L^{2}\left(\Omega^{1} \cup \Omega^{2}\right)\right)^{2}$, the solution $\boldsymbol{z}$ lies in $\left(H^{2}\left(\Omega^{1}\right)\right)^{2} \times\left(H^{2}\left(\Omega^{2}\right)\right)^{2}$ and if there is a constant $C$ so that

$$
\|\boldsymbol{z}\|_{\left(H^{2}\left(\Omega^{1}\right)\right)^{2} \times\left(H^{2}\left(\Omega^{2}\right)\right)^{2}} \leq C\|\boldsymbol{f}\|_{\left(L^{2}\left(\Omega^{1} \cup \Omega^{2}\right)\right)^{2} .} .
$$

Under the latter hypothesis, we are able to obtain this first convergence result in the $L^{2}$-norm for the unilateral contact problem. 
Proposition 6.2. Let $(\boldsymbol{u}, \lambda)$ be the solution of (2.1). Let $\left(\boldsymbol{u}_{h}, \lambda_{h}\right)$ be the solution of (3.4) with $M_{h}=M_{h}^{1,1, *}=M_{h}^{1,2, *}$. Assume that the regularity assumptions of Theorem 4.5 hold true. Suppose that problem (6.3) is regular. Then there is a constant $C(\boldsymbol{u})$ independent of $h$ such that

$$
\left\|\boldsymbol{u}-\boldsymbol{u}_{h}\right\|_{\left(L^{2}\left(\Omega^{1} \cup \Omega^{2}\right)\right)^{2}} \leq C(\boldsymbol{u}) h^{\frac{3}{2}}
$$

Proof. Writing

$$
a\left(\boldsymbol{u}_{h}, \boldsymbol{v}_{h}-\boldsymbol{u}_{h}\right)-L\left(\boldsymbol{v}_{h}-\boldsymbol{u}_{h}\right)=-b\left(\lambda_{h}, \boldsymbol{v}_{h}\right), \quad \forall \boldsymbol{v}_{h} \in \mathbf{V}_{h},
$$

implies that (6.1) is not only true for $\boldsymbol{v}_{h} \in \mathbf{K}_{h}$ but also for $\boldsymbol{v}_{h} \in\left\{\overline{T\left(\mathbf{K}, \boldsymbol{u}_{h}\right)} \cap \mathbf{V}_{h}\right\}+$ $\boldsymbol{u}_{h}$.

Let us choose $\boldsymbol{f}=\boldsymbol{u}-\boldsymbol{u}_{h}$ in 6.3). Invoking (6.2), we can incorporate $\boldsymbol{v}=$ $\boldsymbol{z}+\boldsymbol{u}-\boldsymbol{u}_{h}$ into 6.3 to obtain

$$
\begin{array}{rl}
\left\|\boldsymbol{u}-\boldsymbol{u}_{h}\right\|_{\left(L^{2}\left(\Omega^{1} \cup \Omega^{2}\right)\right)^{2} \leq}^{2} & a\left(\boldsymbol{z}, \boldsymbol{u}-\boldsymbol{u}_{h}\right) \\
= & a\left(\boldsymbol{z}-I_{h} \boldsymbol{z}, \boldsymbol{u}-\boldsymbol{u}_{h}\right)+a\left(I_{h} \boldsymbol{z}, \boldsymbol{u}-\boldsymbol{u}_{h}\right) \\
= & a\left(\boldsymbol{z}-I_{h} \boldsymbol{z}, \boldsymbol{u}-\boldsymbol{u}_{h}\right)+b\left(\lambda_{h}, I_{h} \boldsymbol{z}\right)-b\left(\lambda, I_{h} \boldsymbol{z}+\boldsymbol{u}_{h}\right) \\
& +b\left(\lambda, \boldsymbol{u}_{h}\right) .
\end{array}
$$

It is obvious that $b\left(\lambda, \boldsymbol{u}_{h}\right) \leq 0$. Since $\boldsymbol{z} \in \overline{T\left(\mathbf{K}, \boldsymbol{u}_{h}\right)}$ and $\boldsymbol{z}$ is regular enough, it can be checked that $I_{h} \boldsymbol{z} \in \overline{T\left(\mathbf{K}, \boldsymbol{u}_{h}\right)} \cap \mathbf{V}_{h}$, and thus $b\left(\lambda_{h}, I_{h} \boldsymbol{z}\right) \leq 0$. As a consequence

$$
\left\|\boldsymbol{u}-\boldsymbol{u}_{h}\right\|_{\left(L^{2}\left(\Omega^{1} \cup \Omega^{2}\right)\right)^{2}}^{2} \leq a\left(\boldsymbol{z}-I_{h} \boldsymbol{z}, \boldsymbol{u}-\boldsymbol{u}_{h}\right)-b\left(\lambda, \boldsymbol{z}+\boldsymbol{u}_{h}\right)+b\left(\lambda, \boldsymbol{z}-I_{h} \boldsymbol{z}\right) .
$$

Using $\boldsymbol{z} \in \mathcal{K}$ leads to $b\left(\lambda, \boldsymbol{z}+\boldsymbol{u}_{h}\right) \geq 0$. The remaining terms are handled as previously (as in Lemma 4.2) which gives

$$
\begin{aligned}
\left\|\boldsymbol{u}-\boldsymbol{u}_{h}\right\|_{\left(L^{2}\left(\Omega^{1} \cup \Omega^{2}\right)\right)^{2}}^{2} & \leq C\left\|\boldsymbol{z}-I_{h} \boldsymbol{z}\right\|\left\|\boldsymbol{u}-\boldsymbol{u}_{h}\right\|+b\left(\lambda, \boldsymbol{z}-I_{h} \boldsymbol{z}\right) \\
& \leq C\left(h\left\|\boldsymbol{u}-\boldsymbol{u}_{h}\right\|+h^{\frac{3}{2}}\|\lambda\|_{L^{2}\left(\Gamma_{c}\right)}\right)\|\boldsymbol{z}\|_{\left(H^{2}\left(\Omega^{1}\right)\right)^{2} \times\left(H^{2}\left(\Omega^{2}\right)\right)^{2} .}
\end{aligned}
$$

The regularity of problem (6.3) together with the convergence rate obtained in Theorem 4.5 yields the announced result.

Remark 6.3. The extension of the proposition to $M_{h}=M_{h}^{1,1}=M_{h}^{1,2}$ or $M_{h}=$ $M_{h}^{0,1}=M_{h}^{0,2}$ so as the generalization to nonmatching meshes is an open question actually under consideration.

\section{REFERENCES}

1. R. A. Adams, Sobolev Spaces, Academic Press, 1975. MR 56:9247

2. F. Ben Belgacem, Méthodes d'éléments finis pour des inéquations variationnelles de contact unilatéral. C. R. Acad. Sci. Paris Sér. I Math. 328 (1999), 811-816. MR 2000j:74083

3. F. Ben Belgacem, P. Hild and P. Laborde, Extension of the mortar finite element method to a variational inequality modeling unilateral contact. Math. Models Methods Appl. Sci. 9 (1999), 287-303. MR 99m:73066

4. C. Bernardi, Y. Maday and A. T. Patera, A new nonconforming approach to domain decomposition: the mortar element method, Collège de France Seminar, H. Brezis, J. L. Lions, Pitman, 1994, pp. 13-51. MR 95a:65201

5. F. Brezzi, W. W. Hager and P. A. Raviart, Error Estimates for the Finite Element Solution of Variational Inequalities. Numer. Math. 28 (1977), 431-443. MR 56:7254

6. F. Brezzi, W. W. Hager and P. A. Raviart, Error estimates for the finite element solution of variational inequalities. Part 2: Mixed Methods, Numer. Math. 31 (1978), 1-16. MR 80b:65135 
7. M. D. Canon and C. D. Cullum, A tight upper bound on the rate of convergence of the FrankWolfe algorithm. SIAM J. Control Optim. 6 (1968), 509-516. MR 39:3827] MR 39:2462

8. P. G. Ciarlet, The finite element method for elliptic problems, in Handbook of Numerical Analysis, Volume II, Part 1, Eds. P. G. Ciarlet and J. L. Lions, North Holland, 17-352, 1991. MR 58:25001

9. G. Duvaut and J. L. Lions, Les inéquations en mécanique et en physique, Dunod, Paris, 1972. MR 57:4778

10. M. Frank and P. Wolfe, An algorithm for quadratic programming. Naval Res. Logist. 3 (1956), 95-110.

11. J. Haslinger, I. Hlaváček and J. Nečas, Numerical methods for unilateral problems in solid mechanics, in Handbook of Numerical Analysis, Volume IV, Part 2, Eds. P. G. Ciarlet and J. L. Lions, North Holland, 313-485, 1996. CMP 97:05

12. P. Hild, Numerical Implementation of two nonconforming finite element methods for unilateral contact. Comput. Methods Appl. Mech. Engrg. 184 (2000) 99-123. MR 2000k:74058

13. P. Hild, A propos d'approximation par éléments finis optimale pour les problèmes de contact unilatéral. C. R. Acad. Sci. Paris Sér. I Math. 326 (1998), 1233-1236. MR 99h:65191

14. N. Kikuchi and J. T. Oden, Contact problems in elasticity: a study of variational inequalities and finite element methods, SIAM, Philadelphia, 1988. MR 89j:73097

15. D. Kinderlehrer and G. Stampacchia, An introduction to variational inequalities and their applications, Academic Press, 1980. MR 81g:49013

16. P. Le Tallec and T. Sassi, Domain decomposition with nonmatching grids: augmented lagrangian approach. Math. Comp. 64 (1995), 1367-1396. MR 95m:65212

17. K. Lhalouani and T. Sassi, Nonconforming mixed variational formulation and domain decomposition for unilateral problems. East-West J. Numer. Math. 7 (1999), 23-30. MR 2000b:65217

18. F. Natterer, Optimale $L^{2}-$ Konvergenz finiter Elemente bei Variationsungleichungen. Bonner Math. Schriften 89 (1976), 1-12. MR 56:10070

Laboratoire de Mécanique et CaO, Université de Picardie - Jules Verne, iUt, 48 Rue D'Ostende, 02100 Saint-Quentin, France

E-mail address: patrice.coorevits@insset.u-picardie.fr

Laboratoire de Mathématiques, Université de Savoie / CNRS EP 2067, 73376 Le BourGet Du Lac, France

E-mail address: hild@univ-savoie.fr

Laboratoire de Modélisation Mathématique et de Calcul Scientifique, insa de Lyon

/ CNRS UMR 5585, 20 avenue Albert Einstein, 69621 Villeurbanne, France

E-mail address: khalid@laninsa.insa-lyon.fr

Laboratoire de Modélisation Mathématique et de Calcul Scientifique, insa de Lyon / CNRS UMR 5585, 20 avenue Albert Einstein, 69621 Villeurbanne, France

E-mail address: sassi@laninsa.insa-lyon.fr 